\title{
ON THE GLOBAL BEHAVIOR OF INVERSE MAPPINGS IN TERMS OF PRIME ENDS
}

\section{Nataliya Ilkevych, Evgeny Sevost'yanov and Sergei Skvortsov}

\author{
Zhytomyr Ivan Franko State University \\ 40 Bol'shaya Berdichevskaya Str., 10008 Zhytomyr, Ukraine; ilkevych1980@gmail.com \\ Zhytomyr Ivan Franko State University \\ 40 Bol'shaya Berdichevskaya Str., 10008 Zhytomyr, Ukraine \\ and Institute of Applied Mathematics and Mechanics of NAS of Ukraine, \\ 1 Dobrovol'skogo Str., 84100 Slavyansk, Ukraine; esevostyanov2009@gmail.com \\ Zhytomyr Ivan Franko State University \\ 40 Bol'shaya Berdichevskaya Str., 10008 Zhytomyr, Ukraine; serezha.skv@gmail.com
}

\begin{abstract}
The paper is devoted to the study of mappings with finite distortion, actively studied recently. For mappings whose inverse satisfy the Poletsky inequality, the results on boundary behavior in terms of prime ends are obtained. In particular, it was proved that the families of the indicated mappings are equicontinuous at the points of the boundary if a certain function determining the distortion of the modulus of families of paths under the mappings is integrable.
\end{abstract}

\section{Introduction}

In the theory of quasiconformal mappings, an important place is occupied by the results on their local and boundary behavior, see e.g. [Va Theorem 3.17], [NP 1 , Theorem 3.1], $\left[\mathrm{NP}_{2}\right.$, Theorem 3.1], [Cr, Theorem 8.9] and [MRSY $_{2}$, Theorem 3.1, Corollary 3.6]. Let us mention the following very important result, see $\left[\mathrm{NP}_{1}\right.$, Theorem 3.1].

Theorem. (Näkki-Palka) Let $\mathfrak{F}$ be a family of $K$-quasiconformal mappings of a domain $D \neq \overline{\mathbf{R}^{n}}$ onto a domain $D^{\prime}$ and let either $D$ or $D^{\prime}$ be quasiconformally collared on the boundary. Then $\mathfrak{F}$ is uniformly equicontinuous if and only if each $f \in \mathfrak{F}$ can be extended to a continuous mapping of $\bar{D}$ onto $\overline{D^{\prime}}$ and $\inf _{\mathfrak{F}} q(f(A))>0$ for some continuum $A$ in $D$.

Here $q(f(A))$ denotes the chordal diameter of the set $f(A) \subset \overline{\mathbf{R}^{n}}$, see e.g. [Va, Definition 12.1]. The main goal of this article is to prove results similar to the Näkki-Palka theorem and related to mappings with unbounded characteristic. In this case, we consider domains with a complex structure. We mean that in such domains, mappings may not even have continuous boundary extension in the Euclidean sense. However, under certain additional assumptions, such an extension holds in the sense of the so-called prime ends, to which the present studies relate. Similar studies have taken place in some of our earlier papers, see, for example, [SevSkv $\left.{ }_{1}\right]$ and [Sev]. Unlike previous articles, the main attention here is paid to the behavior of homeomorphisms, the inverse of which satisfy Poletsky-type inequalities. For quasiconformal mappings, consideration of such mappings does not make sense, since the inverse of quasiconformal homeomorphisms belong to the same class. The situation

https://doi.org/10.5186/aasfm.2021.4630

2020 Mathematics Subject Classification: Primary 30C65; Secondary 32U20, 31B15.

Key words: Mappings with a finite and bounded distortion, boundary behaviour, prime ends. 
changes drastically if the characteristic of mappings is unbounded. We will confirm what we said with one of the examples given at the end of this article.

Recall some definitions (see, for example, $\left[\mathrm{KR}_{1}\right]$ and $\left[\mathrm{KR}_{2}\right]$ ). Let $\omega$ be an open set in $\mathbf{R}^{k}, k=1, \ldots, n-1$. A continuous mapping $\sigma: \omega \rightarrow \mathbf{R}^{n}$ is called a $k$-dimensional surface in $\mathbf{R}^{n}$. A surface is an arbitrary $(n-1)$-dimensional surface $\sigma$ in $\mathbf{R}^{n}$. A surface $\sigma$ is called a Jordan surface, if $\sigma(x) \neq \sigma(y)$ for $x \neq y$. In the following, we will use $\sigma$ instead of $\sigma(\omega) \subset \mathbf{R}^{n}, \bar{\sigma}$ instead of $\overline{\sigma(\omega)}$ and $\partial \sigma$ instead of $\overline{\sigma(\omega)} \backslash \sigma(\omega)$. A Jordan surface $\sigma: \omega \rightarrow D$ is called a cut of $D$, if $\sigma$ separates $D$, that is $D \backslash \sigma$ has more than one component, $\partial \sigma \cap D=\varnothing$ and $\partial \sigma \cap \partial D \neq \varnothing$.

A sequence of cuts $\sigma_{1}, \sigma_{2}, \ldots, \sigma_{m}, \ldots$ in $D$ is called $a$ chain, if: (i) the set $\sigma_{m+1}$ is contained in exactly one component $d_{m}$ of the set $D \backslash \sigma_{m}$, wherein $\sigma_{m-1} \subset D \backslash\left(\sigma_{m} \cup\right.$ $\left.d_{m}\right)$; (ii) $\bigcap_{m=1}^{\infty} d_{m}=\varnothing$.

Two chains of cuts $\left\{\sigma_{m}\right\}$ and $\left\{\sigma_{k}^{\prime}\right\}$ are called equivalent, if for each $m=1,2, \ldots$ the domain $d_{m}$ contains all the domains $d_{k}^{\prime}$, except for a finite number, and for each $k=1,2, \ldots$ the domain $d_{k}^{\prime}$ also contains all domains $d_{m}$, except for a finite number.

The end of the domain $D$ is the class of equivalent chains of cuts in $D$. Let $K$ be the end of $D$ in $\mathbf{R}^{n}$, then the set $I(K)=\bigcap_{m=1}^{\infty} \overline{d_{m}}$ is called the impression of the end $K$. Throughout the paper, $\Gamma(E, F, D)$ denotes the family of all paths $\gamma:[a, b] \rightarrow \overline{\mathbf{R}^{n}}$ such that $\gamma(a) \in E, \gamma(b) \in F$ and $\gamma(t) \in D$ for every $t \in[a, b]$. In what follows, $M$ denotes the modulus of a family of paths, and the element $d m(x)$ corresponds to the Lebesgue measure in $\mathbf{R}^{n}, n \geqslant 2$, see [Va $]$. Following [Na], we say that the end $K$ is $a$ prime end, if $K$ contains a chain of cuts $\left\{\sigma_{m}\right\}$ such that $\lim _{m \rightarrow \infty} M\left(\Gamma\left(C, \sigma_{m}, D\right)\right)=0$ for some continuum $C$ in $D$ (see Figure 1 for this).

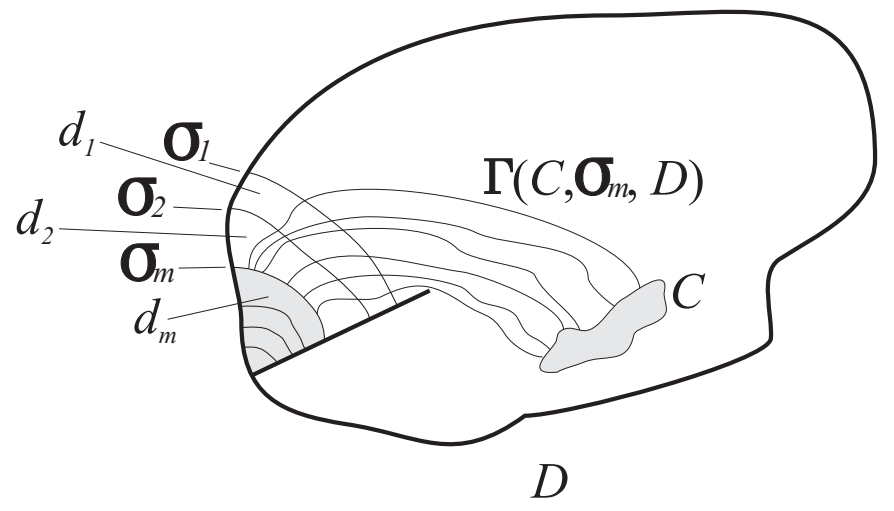

Figure 1. A prime end in some domain.

In the following, the following notation is used: the set of prime ends corresponding to the domain $D$, is denoted by $E_{D}$, and the completion of the domain $D$ by its prime ends is denoted $\bar{D}_{P}$.

Consider the following definition, which goes back to Näkki [Na], see also $\left[\mathrm{KR}_{1}\right]_{-}$ $\left[\mathrm{KR}_{2}\right]$. We say that the boundary of the domain $D$ in $\mathbf{R}^{n}$ is locally quasiconformal, if each point $x_{0} \in \partial D$ has a neighborhood $U$ in $\mathbf{R}^{n}$, which can be mapped by a quasiconformal mapping $\varphi$ onto the unit ball $\mathbf{B}^{n} \subset \mathbf{R}^{n}$ so that $\varphi(\partial D \cap U)$ is the intersection of $\mathbf{B}^{n}$ with the coordinate hyperplane.

Remark 1.1. There are various approaches related to the definition of prime ends. In particular, Näkki's work [Na, Sections 3, 4] uses a definition similar to ours, in which, however, cuts must be connected sets, and the domain must be divided by the cut into exactly two components. For this reason, we note that in domains with 
a locally quasiconformal boundary, the above definition of a prime end is equivalent to our (see [Na, Theorem 4.1] and [IS, Theorem 2.1]).

For a given set $E \subset \mathbf{R}^{n}$, we set $d(E):=\sup _{x, y \in E}|x-y|$. The sequence of cuts $\sigma_{m}, m=1,2, \ldots$, is called regular, if $\overline{\sigma_{m}} \cap \overline{\sigma_{m+1}}=\varnothing$ for $m \in \mathbf{N}$ and, in addition, $d\left(\sigma_{m}\right) \rightarrow 0$ as $m \rightarrow \infty$. If the end $K$ contains at least one regular chain, then $K$ will be called regular. We say that a bounded domain $D$ in $\mathbf{R}^{n}$ is regular, if $D$ can be quasiconformally mapped to a domain with a locally quasiconformal boundary whose closure is a compact in $\mathbf{R}^{n}$, and, besides that, every prime end in $D$ is regular. Note that space $\bar{D}_{P}=D \cup E_{D}$ is metric, which can be demonstrated as follows. If $g$ : $D_{0} \rightarrow D$ is a quasiconformal mapping of a domain $D_{0}$ with a locally quasiconformal boundary onto some domain $D$, then for $x, y \in \bar{D}_{P}$ we put:

$$
\rho(x, y):=\left|g^{-1}(x)-g^{-1}(y)\right|,
$$

where the element $g^{-1}(x), x \in E_{D}$, is to be understood as some (single) boundary point of the domain $D_{0}$. The specified boundary point is unique and well-defined by [IS, Theorem 2.1, Remark 2.1], cf. [Na, Theorem 4.1]. It is easy to verify that $\rho$ in (1.2) is a metric on $\bar{D}_{P}$, and that the topology on $\bar{D}_{P}$, defined by such a method, does not depend on the choice of the map $g$ with the indicated property.

We say that a sequence $x_{m} \in D, m=1,2, \ldots$, converges to a prime end of $P \in E_{D}$ as $m \rightarrow \infty$, if for any $k \in \mathbf{N}$ all elements $x_{m}$ belong to $d_{k}$ except for a finite number. Here $d_{k}$ denotes a sequence of nested domains corresponding to the definition of the prime end $P$. Note that for a homeomorphism of a domain $D$ onto $D^{\prime}$, the end of the domain $D$ uniquely corresponds to some sequence of nested domains in the image under the mapping. See Figure 2 for an illustration.

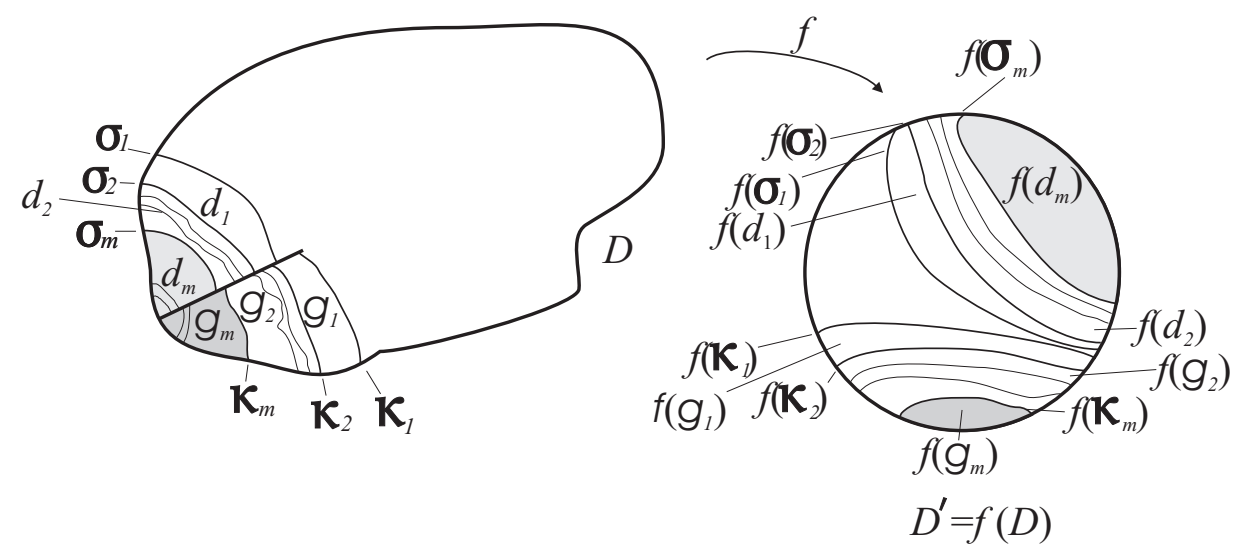

Figure 2. The correspondence between sequences of domains.

Everywhere below, unless otherwise stated, the boundary and the closure of a set are understood in the sense of an extended Euclidean space $\overline{\mathbf{R}^{n}}$. Let $x_{0} \in \bar{D}$, $x_{0} \neq \infty$,

$$
\begin{aligned}
S\left(x_{0}, r\right) & =\left\{x \in \mathbf{R}^{n}:\left|x-x_{0}\right|=r\right\}, \quad S_{i}=S\left(x_{0}, r_{i}\right), \quad i=1,2, \\
A & =A\left(x_{0}, r_{1}, r_{2}\right)=\left\{x \in \mathbf{R}^{n}: r_{1}<\left|x-x_{0}\right|<r_{2}\right\} .
\end{aligned}
$$

Let $Q: \mathbf{R}^{n} \rightarrow \mathbf{R}^{n}$ be a Lebesgue measurable function satisfying the condition $Q(x) \equiv 0$ for $x \in \mathbf{R}^{n} \backslash D$. The mapping $f: D \rightarrow \overline{\mathbf{R}^{n}}$ is called a ring Q-mapping at the point $x_{0} \in \bar{D} \backslash\{\infty\}$, if the condition

$$
M\left(f\left(\Gamma\left(S_{1}, S_{2}, D\right)\right)\right) \leqslant \int_{A \cap D} Q(x) \cdot \eta^{n}\left(\left|x-x_{0}\right|\right) d m(x)
$$


holds for all $0<r_{1}<r_{2}<d_{0}:=\sup _{x \in D}\left|x-x_{0}\right|$ and each Lebesgue measurable function $\eta:\left(r_{1}, r_{2}\right) \rightarrow[0, \infty]$ such that

$$
\int_{r_{1}}^{r_{2}} \eta(r) d r \geqslant 1
$$

The mapping of $f$ is called a ring $Q$-mapping in $D$, if condition (1.3) is satisfied at every point $x_{0} \in D$, and a ring $Q$-mapping in $\bar{D}$, if the condition (1.3) holds at every point $x_{0} \in \bar{D}$. With regard to the definition of such mappings, we point to publications $[\mathrm{RSY}]$ and $\left[\mathrm{MRSY}_{2}\right]$. The class of mappings satisfying relation (1.3) contains itself all conformal and quasiconformal mappings, as well as many mappings with finite distortion, see, for example, [Pol, Theorem 2], [Va 2 , Theorem 3.1] and [MRSY $_{1}$, Theorems 4.6 and 6.10].

Let $(X, d)$ and $\left(X^{\prime}, d^{\prime}\right)$ be metric spaces with distances $d$ and $d^{\prime}$, respectively. A family $\mathfrak{G}$ of mappings $g: X^{\prime} \rightarrow X$ is said to be equicontinuous at a point $y_{0} \in X^{\prime}$, if for every $\varepsilon>0$ there is $\delta=\delta\left(\varepsilon, y_{0}\right)>0$ such that $d\left(g(y), g\left(y_{0}\right)\right)<\varepsilon$ for all $g \in \mathfrak{G}$ and $y \in X^{\prime}$ with $d^{\prime}\left(y, y_{0}\right)<\delta$. The family $\mathfrak{G}$ is equicontinuous if $\mathfrak{G}$ is equicontinuous at every point $y_{0} \in X^{\prime}$.

Everywhere below, unless otherwise stated, $d=\rho$ is one of the metrics in $\bar{D}_{P}$, defined by the relation (1.2), and $d^{\prime}=q$ is a chordal metric defined by formula

$$
q(x, y)=\frac{|x-y|}{\sqrt{1+|x|^{2}} \sqrt{1+|y|^{2}}}, \quad x \neq \infty \neq y, \quad q(x, \infty)=\frac{1}{\sqrt{1+|x|^{2}}} .
$$

For a given set $E \subset \overline{\mathbf{R}^{n}}$, we set

$$
q(E):=\sup _{x, y \in E} q(x, y) .
$$

The quantity $q(E)$ is called the chordal diameter of the set $E$. The boundary of the domain $D$ is called weakly flat at the point $x_{0}$, if for every number $P>0$ and every neighborhood $U$ of this point there is a neighborhood $V$ of point $x_{0}$ such that $M(\Gamma(E, F, D))>P$ for any continua $E$ and $F$, satisfying conditions $F \cap \partial U \neq \varnothing \neq$ $F \cap \partial V$. The boundary of domain $D$ is called weakly flat if it is such at each of its point.

For a given $\delta>0$, domains $D \subset \mathbf{R}^{n}$ and $D^{\prime} \subset \overline{\mathbf{R}^{n}}, n \geqslant 2$, a continuum $A \subset D$ and a Lebesgue measurable function $Q(x): \mathbf{R}^{n} \rightarrow[0, \infty]$ such that $Q(x) \equiv 0$ for $x \notin D$, we denote by $\mathfrak{S}_{\delta, A, Q}\left(D, D^{\prime}\right)$ the family of all homeomorphisms $h$ of $D^{\prime}$ onto $D$ such that the mapping $f=h^{-1}$ satisfies the condition (1.3) in $\bar{D}$, while $q(f(A)) \geqslant \delta$. The following statement is true.

Theorem 1.7. Suppose that $D$ is regular, $D^{\prime}$ has a weakly flat boundary, and any component of $\partial D^{\prime}$ is a non-degenerate continuum. If $Q \in L^{1}(D)$, then each map $h \in \mathfrak{S}_{\delta, A, Q}\left(D, D^{\prime}\right)$ has a continuous extension $\bar{h}:{\overline{D^{\prime}}} \rightarrow \bar{D}_{P}$, in addition, $\bar{h}\left(D^{\prime}\right)=\bar{D}_{P}$, and the family $\mathfrak{S}_{\delta, A, Q}\left(\bar{D}_{P}, \overline{D^{\prime}}\right)$, consisting of all extended mappings $\bar{h}: \overline{D^{\prime}} \rightarrow \bar{D}_{P}$, is equicontinuous in $\overline{D^{\prime}}$.

Remark 1.8. The possibility of continuous extension of a homeomorphism $h$ : $D^{\prime} \rightarrow D$ to the mapping $\bar{h}: \overline{D^{\prime}} \rightarrow \bar{D}_{P}$ in Theorem 1.7 may be established similarly to [GRY, Theorem 6.1]; see also [SalSev, Theorem 2]. Since the proof of this result almost literally repeats the reasoning related to the mentioned publications, we will not give this proof in the present text. 


\section{Preliminaries}

Recall that a path will be called a continuous mapping $\gamma: I \rightarrow \mathbf{R}^{n}$ of a segment, interval or half-interval $I \subset \mathbf{R}$ into $n$-dimensional Euclidean space $\mathbf{R}^{n}$. As usual, the set $|\gamma|=\left\{x \in \mathbf{R}^{n}: \exists t \in I: \gamma(t)=x\right\}$ is called the locus of a path $\gamma: I \rightarrow \mathbf{R}^{n}$. We say that the path $\gamma$ lies in the domain $D$, if its locus belongs to this domain. We also say that paths $\gamma_{1}$ and $\gamma_{2}$ do not intersect each other if their loci do not intersect as sets in $\mathbf{R}^{n}$. By definition, a prime end $P \in E_{D}$ corresponds to a sequence of nested domains $d_{m}, m \geqslant 1$, and if $P \in D$, then we assume that $P$ corresponds to a sequence of balls $B\left(P, r_{m}\right)$ with radii $r_{m} \rightarrow 0$ as $m \rightarrow \infty, r_{m}>0$, which lie in the domain of $D$

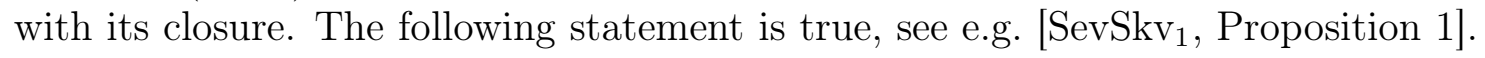

Proposition 2.1. Let $n \geqslant 2$, and let $D$ be a domain in $\mathbf{R}^{n}$ that is locally connected on its boundary. Then every two pairs of points $a \in D, b \in \bar{D}$ and $c \in$ $D, d \in \bar{D}$ can be joined by non-intersecting paths $\gamma_{1}:[0,1] \rightarrow \bar{D}$ and $\gamma_{2}:[0,1] \rightarrow \bar{D}$ so that $\gamma_{i}(t) \in D$ for all $t \in(0,1)$ and all $i=1,2$, while $\gamma_{1}(0)=a, \gamma_{1}(1)=b$, $\gamma_{2}(0)=c$ and $\gamma_{2}(1)=d$.

The proof of the following statement completely repeats the proof of $\left[\mathrm{Va}_{1}\right.$, Theorem 17.10], and therefore is omitted.

Proposition 2.2. Let $D \subset \mathbf{R}^{n}$ be a domain with a locally quasiconformal boundary, then the boundary of this domain is weakly flat. Moreover, the neighborhood of $U$ in the definition of a locally quasiconformal boundary can be taken arbitrarily small, and in this definition $\varphi\left(x_{0}\right)=0$.

The following statement points to the possibility of a "convenient" joining of the points of a regular domain by paths.

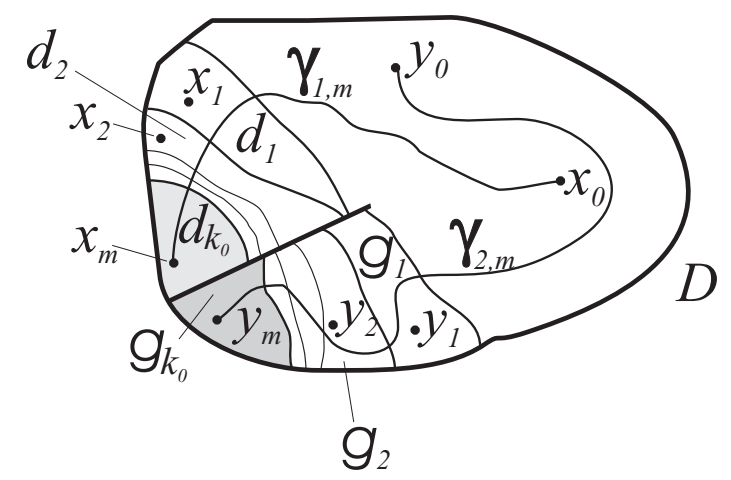

Figure 3. To the statement of Lemma 2.3.

Lemma 2.3. Let $D \subset \mathbf{R}^{n}, n \geqslant 2$, be a regular domain, and let $x_{m} \rightarrow P_{1}$, $y_{m} \rightarrow P_{2}$ as $m \rightarrow \infty, P_{1}, P_{2} \in \bar{D}_{P}, P_{1} \neq P_{2}$. Suppose that $d_{m}, g_{m}, m=1,2, \ldots$, are sequences of descending domains, corresponding to $P_{1}$ and $P_{2}, d_{1} \cap g_{1}=\varnothing$, and $x_{0}, y_{0} \in D \backslash\left(d_{1} \cup g_{1}\right)$. Then there are arbitrarily large $k_{0} \in \mathbf{N}, M_{0}=M_{0}\left(k_{0}\right) \in \mathbf{N}$ and $0<t_{1}=t_{1}\left(k_{0}\right), t_{2}=t_{2}\left(k_{0}\right)<1$ for which the following condition is fulfilled: for each $m \geqslant M_{0}$ there are non-intersecting paths

$$
\gamma_{1, m}(t)=\left\{\begin{array}{ll}
\widetilde{\alpha}(t), & t \in\left[0, t_{1}\right], \\
\widetilde{\alpha_{m}}(t), & t \in\left[t_{1}, 1\right],
\end{array} \gamma_{2, m}(t)= \begin{cases}\widetilde{\beta}(t), & t \in\left[0, t_{2}\right], \\
\widetilde{\beta_{m}}(t), & t \in\left[t_{2}, 1\right],\end{cases}\right.
$$

such that:

1) $\gamma_{1, m}(0)=x_{0}, \gamma_{1, m}(1)=x_{m}, \gamma_{2, m}(0)=y_{0}$ and $\gamma_{2, m}(1)=y_{m}$; 
2) $\left|\gamma_{1, m}\right| \cap \overline{g_{k_{0}}}=\varnothing=\left|\gamma_{2, m}\right| \cap \overline{d_{k_{0}}}$;

3) $\widetilde{\alpha_{m}}(t) \in d_{k_{0}+1}$ for $t \in\left[t_{1}, 1\right]$ and $\widetilde{\beta_{m}}(t) \in g_{k_{0}+1}$ for $t \in\left[t_{2}, 1\right]$ (see Figure 3 ).

Proof. Since, by condition, $D$ is a regular domain, it can be mapped onto some domain with a locally quasiconformal boundary by (some) quasiconformal mapping $h: D \rightarrow D_{0}$. Note that the domain $D_{0}$ is locally connected on its boundary, which follows directly from the definition of local quasiconformality.

Note that, if $P_{1}$ and $P_{2}$ are different prime ends in $D$, then $h\left(P_{1}\right)$ and $h\left(P_{2}\right)$ are different prime ends in $D_{0}$. Indeed, let $\sigma_{m}$ be a sequence of cuts corresponding to the prime end $P_{1}$. The fact that $h\left(\sigma_{m}\right)$ is also a cut of the domain $D_{0}$ is obvious, since $h$ is a homeomorphism. Now we verify that the sequence $h\left(\sigma_{m}\right), m=1,2, \ldots$, is a chain. The conditions (ii) and (iii) taken from the definition of a chain are obvious, since $h$ is a homeomorphism. We now verify the condition (i): $\overline{h\left(\sigma_{m}\right)} \cap \overline{h\left(\sigma_{m+1}\right)}=\varnothing$ for $m \in \mathbf{N}$. Suppose the contrary, namely, that $\overline{h\left(\sigma_{m}\right)} \cap \overline{h\left(\sigma_{m+1}\right)} \neq \varnothing$ at least for one $m \in \mathbf{N}$. Then there is a point $x_{0} \in \partial D_{0}$ such that $x_{0} \in \overline{h\left(\sigma_{m}\right)} \cap \overline{h\left(\sigma_{m+1}\right)}$. Proposition 2.2 implies that $M\left(\Gamma\left(h\left(\sigma_{m}\right), h\left(\sigma_{m+1}\right), D_{0}\right)\right)=\infty$. On the other hand, in view of the definition of the modulus of families of paths, $M\left(\Gamma\left(\sigma_{m}, \sigma_{m+1}, D\right)\right) \leqslant$ $l_{0}^{-n} \cdot m(D)<\infty$, where $l_{0}:=\operatorname{dist}\left(\sigma_{m}, \sigma_{m+1}\right)>0$ and $m(D)$ is a Lebesgue measure of $D$. Here it was also taken into account that the domain $D$ is bounded, so that $m(D)<\infty$. Then, due to the quasiconformality of the mapping $h$, we have that $M\left(\Gamma\left(h\left(\sigma_{m}\right), h\left(\sigma_{m+1}\right), D_{0}\right)\right) \leqslant K \cdot M\left(\Gamma\left(\sigma_{m}, \sigma_{m+1}, D_{0}\right)\right)<\infty$, where $K<\infty$ is some constant. The resulting contradiction refutes the assumption that $\overline{h\left(\sigma_{m}\right)} \cap \overline{h\left(\sigma_{m+1}\right)} \neq$ $\varnothing$.

Thus, the chain of cuts $h\left(\sigma_{m}\right)$ defines some end $h\left(P_{1}\right)$. The fact that this end is prime also simply follows from the quasiconformality of the mapping $h$. Similarly, $h\left(P_{2}\right)$ is a prime end in $D_{0}$.

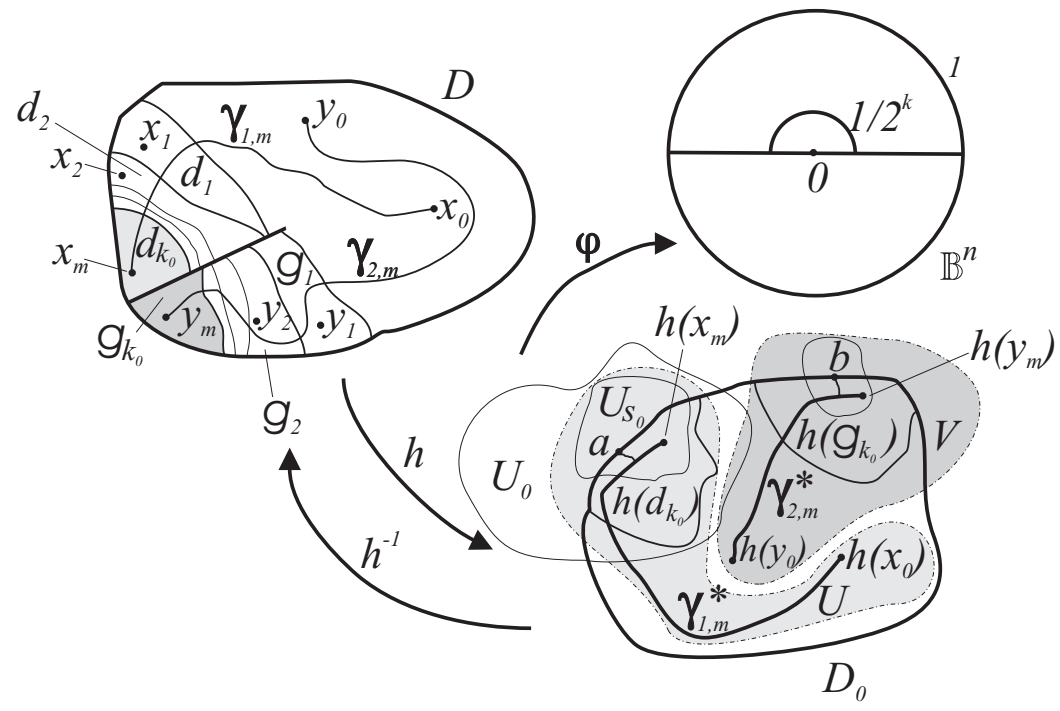

Figure 4. To the proof of Lemma 2.3.

Note that the impressions $I\left(h\left(P_{1}\right)\right)$ and $I\left(h\left(P_{2}\right)\right)$ of $h\left(P_{1}\right)$ and $h\left(P_{2}\right)$ are some different points $a$ and $b$ in $\partial D_{0}$ (see [IS, Lemma 2.2], cf. [Na, Theorem 4.1]). If $P_{1}$ or $P_{2}$ are inner points of $D$, then $h\left(P_{1}\right)$ (or $h\left(P_{2}\right)$ ) are inner points of $D_{0}$, which we denote by $a$ or $b$, respectively. Since by assumption $x_{0}, y_{0} \in D \backslash\left(d_{1} \cup g_{1}\right)$, then, in particular, $P_{1} \neq x_{0} \neq P_{2}, P_{1} \neq y_{0} \neq P_{2}$. This implies that $a, b, h\left(x_{0}\right)$ and $h\left(y_{0}\right)$ are four different points in $\overline{D_{0}}$, at least two of which are inner points of $D_{0}$ (see Figure 4 ). 
By Proposition 2.1, one can join pairs of points $a, h\left(x_{0}\right)$ and $b, h\left(y_{0}\right)$ by disjoint paths $\alpha:[0,1] \rightarrow \overline{D_{0}}$ and $\beta:[0,1] \rightarrow \overline{D_{0}}$ so that $|\alpha| \cap|\beta|=\varnothing, \alpha(t), \beta(t) \in D_{0}$ for all $t \in(0,1), \alpha(0)=h\left(x_{0}\right), \alpha(1)=a, \beta(0)=h\left(y_{0}\right)$ and $\beta(1)=b$. Since $\mathbf{R}^{n}$ is a normal topological space, the loci $|\alpha|$ and $|\beta|$ have non-intersecting open neighborhoods $U, V$ such that

$$
|\alpha| \subset U, \quad|\beta| \subset V
$$

Here two cases are possible: either $h\left(P_{1}\right)$ is a prime end in $E_{D_{0}}$, or a point in $D_{0}$. Let $h\left(P_{1}\right)$ be a prime end in $E_{D_{0}}$. Since $I\left(h\left(P_{1}\right)\right)=a$, then there is a number $k_{1} \in \mathbf{N}$ such that $\overline{h\left(d_{k}\right)} \subset U$ for $k \geqslant k_{1}$. If $h\left(P_{1}\right)$ is a point of $D$, then there is also a number $k_{1} \in \mathbf{N}$ such that $\overline{h\left(d_{k}\right)} \subset U$ for all $k \geqslant k_{1}$, where $d_{k}:=B\left(P_{1}, r_{k}\right), r_{k} \rightarrow 0$ as $k \rightarrow \infty$ and $r_{k}>0$. In either of these two cases, $\overline{h\left(d_{k}\right)} \subset U$ for $k \geqslant k_{1}$. Similarly, there is a number $k_{2} \in \mathbf{N}$ such that $\overline{h\left(g_{k}\right)} \subset V$ for all $k \geqslant k_{2}$. Then for $k_{0}:=\max \left\{k_{1}, k_{2}\right\}$ we obtain that

$$
\overline{h\left(d_{k}\right)} \subset U, \quad \overline{h\left(g_{k}\right)} \subset V, \quad U \cap V=\varnothing, \quad k \geqslant k_{0} .
$$

Since the sequence $x_{m}$ converges to $P_{1}$ as $m \rightarrow \infty$, then $h\left(x_{m}\right)$ converges to $a$. Therefore, there is a number $m_{1} \in \mathbf{N}$ such that $h\left(x_{m}\right) \in h\left(d_{k_{0}+1}\right)$ for $m \geqslant m_{1}$. Similarly, since $y_{m}$ converges to $P_{2}$ as $m \rightarrow \infty$, then $h\left(y_{m}\right)$ converges to $b$. Therefore, there is a number $m_{2} \in \mathbf{N}$ such that $h\left(y_{m}\right) \in h\left(g_{k_{0}+1}\right)$ for $m \geqslant m_{2}$. Put $M_{0}:=$ $\max \left\{m_{1}, m_{2}\right\}$. Show that

$$
|\alpha| \cap h\left(d_{k_{0}+1}\right) \neq \varnothing, \quad|\beta| \cap h\left(g_{k_{0}+1}\right) \neq \varnothing .
$$

It suffices to establish the first of these relations, since the second relation can be proved similarly. If $a=h\left(P_{1}\right)$ is an inner point of $D_{0}$, then this inclusion is obvious. Now suppose that $h\left(P_{1}\right)$ is a prime end in $E_{D_{0}}$. Since the domain $D_{0}$ has a locally quasiconformal boundary, there is a sequence of spheres $S\left(0,1 / 2^{k}\right), k=0,1,2, \ldots$, a decreasing sequence of neighborhoods $U_{k}$ of the point $a$ and some quasiconformal mapping $\varphi: U_{0} \rightarrow \mathbf{B}^{n}$, for which $\varphi\left(U_{k}\right)=B\left(0,1 / 2^{k}\right), \varphi\left(\partial U_{k} \cap D_{0}\right)=S\left(0,1 / 2^{k}\right) \cap \mathbf{B}_{+}^{n}$, where $\mathbf{B}_{+}^{n}=\left\{x=\left(x_{1}, \ldots, x_{n}\right):|x|<1, x_{n}>0\right\}$ (see the arguments given in the proof of [Na, Lemma 3.5]). Note that $U_{k} \cap D_{0}$ is a domain, since $U_{k} \cap D_{0}=\varphi^{-1}\left(B_{+}\left(0,1 / 2^{k}\right)\right)$, $B_{+}\left(0,1 / 2^{k}\right)=\left\{x=\left(x_{1}, \ldots, x_{n}\right):|x|<1 / 2^{k}, x_{n}>0\right\}$, and $\varphi$ is a homeomorphism. In addition, the sequence of domains $U_{k} \cap D_{0}$ corresponds to some prime end, the impression of which is the point $a$, and the corresponding cuts are the sets $\sigma_{k}:=$ $\partial U_{k} \cap D_{0}$. By [Na, Theorem 4.1] and Remark 1.1, the point $a \in D_{0}$ corresponds to exactly one prime end, therefore every domain $h\left(d_{m}\right)$ contains all domains $U_{k} \cap D_{0}$, except for a finite number, and vice versa. In particular, there is $s_{0} \in \mathbf{N}$ such that $U_{k} \cap D_{0} \subset h\left(d_{k_{0}+1}\right)$ for all $k \geqslant s_{0}$. Since $a \in|\alpha|$, there is $t_{1} \in(0,1)$ such that $p:=\alpha\left(t_{1}\right) \in U_{s_{0}} \cap D_{0}$. But then also $p \in h\left(d_{k_{0}+1}\right)$, since $U_{s_{0}} \cap D_{0} \subset h\left(d_{k_{0}+1}\right)$. The first relation in (2.6) is proved. As we said above, the second relation may be proved in exactly the same way.

So, let $p:=\alpha\left(t_{1}\right) \in|\alpha| \cap h\left(d_{k_{0}+1}\right)$. Fix $m \geqslant M_{0}$ and join the point $p$ with the point $h\left(x_{m}\right)$ using the path $\alpha_{m}:\left[t_{1}, 1\right] \rightarrow h\left(d_{k_{0}+1}\right)$ so that $\alpha_{m}\left(t_{1}\right)=p, \alpha_{m}(1)=h\left(x_{m}\right)$, what is possible because $h\left(d_{k_{0}+1}\right)$ is a domain. Set

$$
\gamma_{1, m}^{*}(t)= \begin{cases}\alpha(t), & t \in\left[0, t_{1}\right], \\ \alpha_{m}(t), & t \in\left[t_{1}, 1\right] .\end{cases}
$$

Note that the path $\gamma_{1, m}^{*}$ completely lies in $U$. Reasoning similarly, we have the point $t_{2} \in(0,1)$ and the point $q:=\beta\left(t_{2}\right) \in|\beta| \cap h\left(g_{k_{0}+1}\right)$. Fix $m \geqslant M_{0}$ and join the 
point $q$ with the point $h\left(y_{m}\right)$ using the path $\beta_{m}:\left[t_{2}, 1\right] \rightarrow h\left(g_{k_{0}+1}\right)$ so that $\beta_{m}\left(t_{2}\right)=q$, $\beta_{m}(1)=h\left(y_{m}\right)$, that is possible, because $h\left(g_{k_{0}+1}\right)$ is a domain. Set

$$
\gamma_{2, m}^{*}(t)= \begin{cases}\beta(t), & t \in\left[0, t_{2}\right], \\ \beta_{m}(t), & t \in\left[t_{2}, 1\right] .\end{cases}
$$

Note that the path $\gamma_{2, m}^{*}$ completely lies in $V$. Set

$$
\gamma_{1, m}:=h^{-1}\left(\gamma_{1, m}^{*}\right), \quad \gamma_{2, m}:=h^{-1}\left(\gamma_{2, m}^{*}\right) .
$$

Note that the paths $\gamma_{1, m}$ and $\gamma_{2, m}$ satisfy all the conditions of Lemma 2.3 for $m \geqslant M_{0}$. In fact, by definition, these paths join the points $x_{m}, x_{0}$ and $y_{m}, y_{0}$, respectively. The paths $\gamma_{1, m}$ and $\gamma_{2, m}$ do not intersect, since their images under the mapping $h$ belong to non-intersecting neighborhoods $U$ and $V$, respectively.

Note also that $\left|\gamma_{1, m}\right| \cap \overline{g_{k_{0}}}=\varnothing$ for $m \geqslant M_{0}$. Indeed, if $x \in\left|\gamma_{1, m}\right| \cap \overline{g_{k_{0}}}$, then either $x \in\left|\gamma_{1, m}\right| \cap g_{k_{0}}$ or $x \in\left|\gamma_{1, m}\right| \cap \partial g_{k_{0}}$. In the first case, if $x \in\left|\gamma_{1, m}\right| \cap g_{k_{0}}$ then $h(x) \in\left|\gamma_{1, m}^{*}\right| \cap h\left(g_{k_{0}}\right) \subset U \cap h\left(g_{k_{0}}\right)$, which is impossible due to the relation (2.5). In the second case, if $x \in\left|\gamma_{1, m}\right| \cap \partial g_{k_{0}}$, then there is a sequence $z_{m} \in g_{k_{0}}$ such that $z_{m} \rightarrow x$ as $m \rightarrow \infty$. Now $h\left(z_{m}\right) \rightarrow h(x)$ as $m \rightarrow \infty$ and, therefore, $h(x) \in \overline{h\left(g_{k_{0}}\right)}$. At the same time, $h(x) \in U$, and this is impossible by virtue of relation (2.5). Thus, the relation $\left|\gamma_{1, m}\right| \cap \overline{g_{k_{0}}}=\varnothing$ for $m \geqslant M_{0}$ is established.

Similarly, $\left|\gamma_{2, m}\right| \cap \overline{d_{k_{0}}}=\varnothing$ for $m \geqslant M_{0}$. Finally, defining paths $\widetilde{\alpha}, \widetilde{\alpha}_{m}, \widetilde{\beta}$ and $\widetilde{\beta}_{m}$ by means of relations $\widetilde{\alpha}=h^{-1}(\alpha), \widetilde{\alpha}_{m}=h^{-1}\left(\alpha_{m}\right), \widetilde{\beta}=h^{-1}(\beta)$ and $\widetilde{\beta}_{m}=h^{-1}\left(\beta_{m}\right)$, we see that these paths correspond to the construction of $\gamma_{1, m}$ and $\gamma_{2, m}$, and also satisfy conditions 3 ) from the formulation of the lemma. Lemma 2.3 is proved.

Consider the family of paths joining $\left|\gamma_{1, m}\right|$ and $\left|\gamma_{2, m}\right|$ from the previous lemma. The following statement contains the upper estimate of the modulus of the transformed family of paths under the mapping $f$ with the inequality (1.3).

Lemma 2.10. Let $D \subset \mathbf{R}^{n}, n \geqslant 2$, be a regular domain in $\mathbf{R}^{n}$, and let $f: D \rightarrow$ $\overline{\mathbf{R}^{n}}$ be a continuous map satisfying the estimate (1.3) at every point $x_{0} \in \bar{D}$ and some $Q \in L^{1}(D)$. Then, under the conditions and notation of Lemma 2.3, it is possible to choose the sequence of domains $d_{m}$ and the number $k_{0}$ in such a way that there exist a constant $0<N=N\left(k_{0}, Q, D\right)<\infty$, independent of the parameter $m$ and a mapping $f$, under which

$$
M\left(f\left(\Gamma\left(\left|\gamma_{1, m}\right|,\left|\gamma_{2, m}\right|, D\right)\right)\right) \leqslant N, \quad m \geqslant M_{0}=M_{0}\left(k_{0}\right) .
$$

Proof. By [IS, Lemma 3.1], cf. [KR 2 , Lemma 1], the prime end $P_{1}$ contains a chain of cuts $\sigma_{m}$ lying on spheres $S\left(\overline{x_{0}}, r_{m}\right)$ such that $\overline{x_{0}} \in \partial D$ and $r_{m} \rightarrow 0$ as $m \rightarrow \infty$. Let $d_{m}$ be a sequence of domains corresponding to cuts $\sigma_{m}$. Consider $M_{0}=M_{0}\left(k_{0}\right)$ and paths $\gamma_{1, m}$ and $\gamma_{2, m}$ corresponding to this number.

Using the notation of Lemma 2.3, we put

$$
\varepsilon_{0}:=\min \left\{\operatorname{dist}\left(|\widetilde{\alpha}|, \overline{g_{k_{0}}}\right), \operatorname{dist}(|\widetilde{\alpha}|,|\widetilde{\beta}|)\right\}>0 .
$$

Now, consider covering of $|\widetilde{\alpha}|$ of the following type: $\bigcup_{x \in|\widetilde{\alpha}|} B\left(x, \varepsilon_{0} / 4\right)$. Since $|\widetilde{\alpha}|$ is compact in $D$, there are $i_{1}, \ldots, i_{N_{0}}$ such that $|\widetilde{\alpha}| \subset \bigcup_{i=1}^{N_{0}} B\left(z_{i}, \varepsilon_{0} / 4\right)$, where $z_{i} \in|\widetilde{\alpha}|$ for $1 \leqslant i \leqslant N_{0}$. Taking into account [Ku, Theorem 1.I.5.46], it is easy to verify that

$$
\Gamma\left(|\widetilde{\alpha}|,\left|\gamma_{2, m}\right|, D\right) \subset \bigcup_{i=1}^{N_{0}} \Gamma\left(S\left(z_{i}, \varepsilon_{0} / 4\right), S\left(z_{i}, \varepsilon_{0} / 2\right), D\right) .
$$


Putting

$$
\eta(t)= \begin{cases}4 / \varepsilon_{0}, & t \in\left[\varepsilon_{0} / 4, \varepsilon_{0} / 2\right], \\ 0, & t \notin\left[\varepsilon_{0} / 4, \varepsilon_{0} / 2\right],\end{cases}
$$

we observe that the function $\eta$ satisfies relation (1.4). Then, by the definition of a ring $Q$-map in (1.3) and taking into account the semi-additivity of the modulus of families of paths, see [ $\mathrm{Va}_{1}$, Theorem 6.2], we obtain that

$$
\begin{aligned}
M\left(f\left(\Gamma\left(|\widetilde{\alpha}|,\left|\gamma_{2, m}\right|, D\right)\right)\right) & \leqslant \sum_{i=1}^{N_{0}} M\left(f\left(\Gamma\left(S\left(z_{i}, \varepsilon_{0} / 4\right), S\left(z_{i}, \varepsilon_{0} / 2\right), D\right)\right)\right) \\
& \leqslant \frac{N_{0} 4^{n}\|Q\|_{1}}{\varepsilon_{0}^{n}}, \quad m \geqslant M_{0},
\end{aligned}
$$

where $\|Q\|_{1}=\int_{D} Q(x) d m(x)$. Further, by [Ku, Theorem 1.I.5.46], we obtain that

$$
\Gamma\left(\left|\widetilde{\alpha_{m}}\right|,\left|\gamma_{2, m}\right|, D\right) \leqslant \Gamma\left(S\left(\overline{x_{0}}, r_{k_{1}}\right), S\left(\overline{x_{0}}, r_{k_{2}}\right), D\right) .
$$

Arguing as above, choosing an admissible function

$$
\eta(t)= \begin{cases}1 /\left(r_{k_{0}}-r_{k_{0}+1}\right), & t \in\left[r_{k_{0}+1}, r_{k_{0}}\right], \\ 0, & t \notin\left[r_{k_{0}+1}, r_{k_{0}}\right],\end{cases}
$$

we obtain that

$$
\begin{aligned}
M\left(f\left(\Gamma\left(\left|\widetilde{\alpha_{m}}\right|,\left|\gamma_{2, m}\right|, D\right)\right)\right) & \leqslant M\left(f\left(\Gamma\left(S\left(\overline{x_{0}}, r_{k_{0}}\right), S\left(\overline{x_{0}}, r_{k_{0}+1}\right), D\right)\right)\right) \\
& \leqslant \frac{\|Q\|_{1}}{\left(r_{k_{0}}-r_{k_{0}+1}\right)^{n}}, \quad m \geqslant M_{0} .
\end{aligned}
$$

Now note that

$$
\Gamma\left(\left|\gamma_{1, m}\right|,\left|\gamma_{2, m}\right|, D\right) \subset \Gamma\left(\left|\widetilde{\alpha_{m}}\right|,\left|\gamma_{2, m}\right|, D\right) \cup \Gamma\left(|\widetilde{\alpha}|,\left|\gamma_{2, m}\right|, D\right) .
$$

In this case, from (2.11) and (2.12), taking into account the semi-additivity of the modulus of families of paths, we obtain that

$$
M\left(f\left(\Gamma\left(\left|\gamma_{1, m}\right|,\left|\gamma_{2, m}\right|, D\right)\right)\right) \leqslant\left(\frac{N_{0} 4^{n}}{\varepsilon_{0}^{n}}+\frac{1}{\left(r_{k_{0}}-r_{k_{0}+1}\right)^{n}}\right)\|Q\|_{1}, \quad m \geqslant M_{0} .
$$

The right side of the last relation does not depend on $m$, so that we can put $N:=$ $\left(\frac{N_{0} 4^{n}}{\varepsilon_{0}^{n}}+\frac{1}{\left(r_{k_{0}}-r_{k_{0}+1}\right)^{n}}\right)\|Q\|_{1}$. Lemma 2.10 is completely proved.

The following statement indicates that for some wide class of mappings fixing the diameter of the image of a certain non-degenerate continuum, the image of this continuum cannot be close to the boundary of the corresponding domain under these mappings. Note that similar statements were previously known for quasiconformal mappings, see, for example, [Va 1 , Theorems 21.13 and 21.14]. We may also point to our recent result on this, see [SevSkv 1 , Lemma 2(v)] and [SevSkv 2 , Lemma 4.1].

Lemma 2.13. Let $n \geqslant 2$, let $D$ be a regular domain in $\mathbf{R}^{n}$, and let $D^{\prime}$ be some domain in $\overline{\mathbf{R}^{n}}$. Suppose that $D^{\prime}$ has a weakly flat boundary, $Q \in L^{1}(D)$ and, moreover, any connected component of $\partial D^{\prime}$ does not degenerate into a point. Let $f_{m}: D \rightarrow D^{\prime}$ be a sequence of homeomorphisms of $D$ onto $D^{\prime}$, satisfying the relation (1.3) in $D$ with the same function $Q$. Suppose also that there is a continuum $A \subset D$ and a number $\delta>0$ such that $q\left(f_{m}(A)\right) \geqslant \delta>0$ for all $m=1,2, \ldots$, where, as usual, $q\left(f_{m}(A)\right)$ is defined by (1.6). Then there is $\delta_{1}>0$ such that

$$
q\left(f_{m}(A), \partial D^{\prime}\right)>\delta_{1}>0 \quad \forall m \in \mathbf{N},
$$


where $q\left(f_{m}(A), \partial D^{\prime}\right)=\inf _{x \in f_{m}(A), y \in \partial D^{\prime}} q(x, y)$.

Proof. We carry out the proof by contradiction. Suppose that the conclusion of the lemma is not true. Then for each $k \in \mathbf{N}$ there is some number $m=m_{k}$ such that $q\left(f_{m_{k}}(A), \partial D^{\prime}\right)<1 / k$. Of course, we may assume that the sequence $m_{k}$ increases on $k$. Since $\overline{\mathbf{R}^{n}}$ is compact, the set $\partial D^{\prime}$ is also compact in extended Euclidean space. Note that the set $f_{m_{k}}(A)$ is compact as a continuous image of a compact set $A \subset D$ under the mapping $f_{m_{k}}$. In this case, there are elements $x_{k} \in f_{m_{k}}(A)$ and $y_{k} \in \partial D^{\prime}$ such that $q\left(f_{m_{k}}(A), \partial D^{\prime}\right)=q\left(x_{k}, y_{k}\right)<1 / k($ see Figure 5$)$.

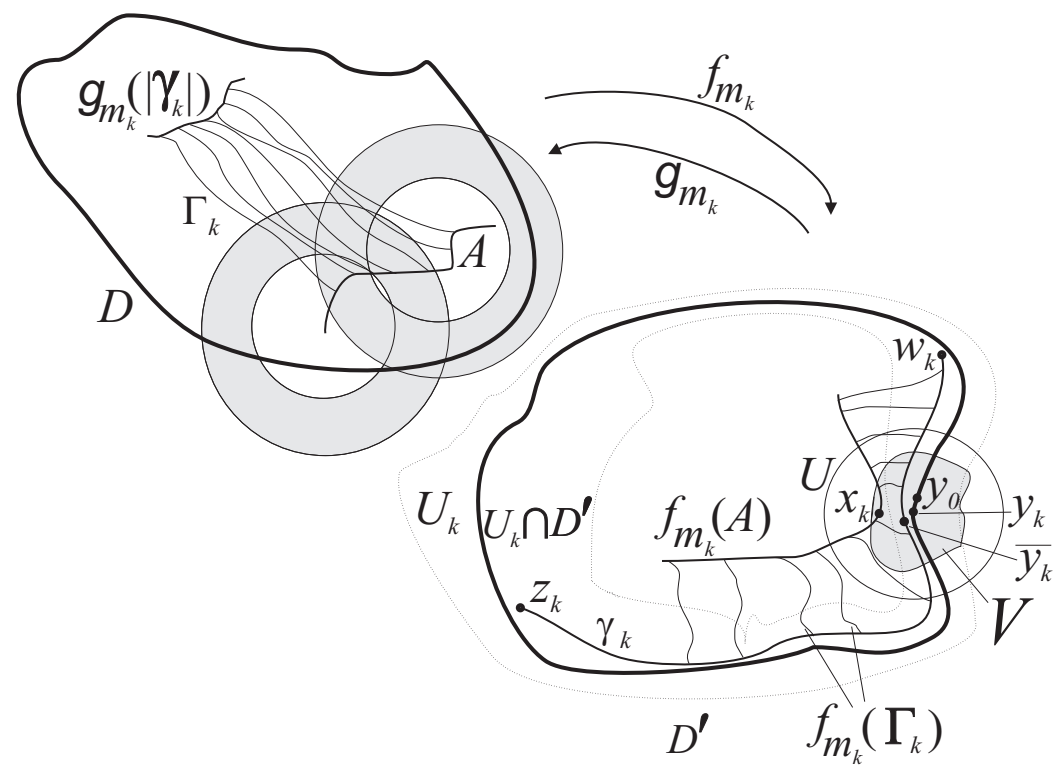

Figure 5. To the proof of Lemma 2.13.

Since $\partial D^{\prime}$ is a compact set, we may assume that $y_{k} \rightarrow y_{0} \in \partial D^{\prime}$ as $k \rightarrow \infty$; then also

$$
x_{k} \rightarrow y_{0} \in \partial D^{\prime}, \quad k \rightarrow \infty .
$$

Let $K_{0}$ be a connected component of the set $\partial D^{\prime}$, containing $y_{0}$. Obviously, $K_{0}$ is a nondegenerate continuum in $\overline{\mathbf{R}^{n}}$. Since $D^{\prime}$ has a weakly flat boundary, the mapping $g_{m_{k}}:=f_{m_{k}}^{-1}$ can be extended to a continuous mapping $\bar{g}_{m_{k}}: \overline{D^{\prime}} \rightarrow \bar{D}_{P}$ (see Remark 1.8). Moreover, $\bar{g}_{m_{k}}$ is uniformly continuous on the set $\overline{D^{\prime}}$ for every fixed $k$, because the mapping $\bar{g}_{m_{k}}$ is continuous on the compact set $\overline{D^{\prime}}$. Let $\rho$ be one of the metrics in $\bar{D}_{P}$, defined in (1.2), and let $g: D_{0} \rightarrow D$ be a quasiconformal mapping of some domain $D_{0}$ with locally quasiconformal boundary corresponding to the definition of the metric $\rho$ in (1.2). In this case, for each $\varepsilon>0$ there is $\delta_{k}=\delta_{k}(\varepsilon)<1 / k$ such that

$$
\rho\left(\bar{g}_{m_{k}}(x), \bar{g}_{m_{k}}\left(x_{0}\right)\right)<\varepsilon \quad \forall x, x_{0} \in \overline{D^{\prime}}, \quad q\left(x, x_{0}\right)<\delta_{k}, \quad \delta_{k}<1 / k .
$$

Choose $\varepsilon>0$ such that

$$
\varepsilon<(1 / 2) \cdot \operatorname{dist}\left(\partial D_{0}, g^{-1}(A)\right),
$$

where $A$ is a continuum from the conditions of the lemma. Denote $B_{q}\left(x_{0}, r\right)=\{x \in$ $\left.\overline{\mathbf{R}^{n}}: q\left(x, x_{0}\right)<r\right\}$. For a given $k \in \mathbf{N}$, we set

$$
B_{k}:=\bigcup_{x_{0} \in K_{0}} B_{q}\left(x_{0}, \delta_{k}\right), \quad k \in \mathbf{N}
$$


Since the set $B_{k}$ is a neighborhood of the continuum $K_{0}$, due to [HK, Lemma 2.2] there is a neighborhood $U_{k}$ of the set $K_{0}$ such that $U_{k} \subset B_{k}$ and $U_{k} \cap D^{\prime}$ is connected. Without loss of generality, we may assume that $U_{k}$ is an open set, so $U_{k} \cap D^{\prime}$ is also path connected (see $\left[\mathrm{MRSY}_{3}\right.$, Proposition 13.1]). Let $q\left(K_{0}\right)=m_{0}$, where the chordal diameter $q\left(K_{0}\right)$ of the set $K_{0}$ is defined by the relation (1.6). In this case, there are $z_{0}, w_{0} \in K_{0}$ such that $q\left(K_{0}\right)=q\left(z_{0}, w_{0}\right)=m_{0}$. So, there are sequences $\overline{y_{k}} \in U_{k} \cap D^{\prime}$, $z_{k} \in U_{k} \cap D^{\prime}$ and $w_{k} \in U_{k} \cap D^{\prime}$ such that $z_{k} \rightarrow z_{0}, \overline{y_{k}} \rightarrow y_{0}$ and $w_{k} \rightarrow w_{0}$ as $k \rightarrow \infty$. We may assume that

$$
q\left(z_{k}, w_{k}\right)>m_{0} / 2 \quad \forall k \in \mathbf{N} .
$$

Since the set $U_{k} \cap D^{\prime}$ is path-connected, we can sequentially join the points $z_{k}, \overline{y_{k}}$ and $w_{k}$ using some path $\gamma_{k}$ in $U_{k} \cap D^{\prime}$. As usual, we denote by $\left|\gamma_{k}\right|$ the locus of the path $\gamma_{k}$ in the domain $D^{\prime}$. Then $g_{m_{k}}\left(\left|\gamma_{k}\right|\right)$ is a compact set in the domain $D$. If $x \in\left|\gamma_{k}\right|$, then there is $x_{0} \in K_{0}$ such that $x \in B\left(x_{0}, \delta_{k}\right)$. Put $\omega \in A \subset D$. Since $x \in\left|\gamma_{k}\right|$ and, moreover, $x$ is an inner point of the domain $D^{\prime}$, we can write here $g_{m_{k}}(x)$ instead of $\bar{g}_{m_{k}}(x)$. By the relations (2.14) and (2.15), as well as by the triangle inequality, we obtain that for sufficiently large $k \in \mathbf{N}$,

$$
\begin{aligned}
\rho\left(g_{m_{k}}(x), \omega\right) & \geqslant \rho\left(\omega, \bar{g}_{m_{k}}\left(x_{0}\right)\right)-\rho\left(\bar{g}_{m_{k}}\left(x_{0}\right), g_{m_{k}}(x)\right) \\
& \geqslant \operatorname{dist}\left(\partial D_{0}, g^{-1}(A)\right)-(1 / 2) \cdot \operatorname{dist}\left(\partial D_{0}, g^{-1}(A)\right) \\
& =(1 / 2) \cdot \operatorname{dist}\left(\partial D_{0}, g^{-1}(A)\right)>\varepsilon,
\end{aligned}
$$

where $\operatorname{dist}\left(\partial D_{0}, g^{-1}(A)\right):=\inf _{x \in \partial D_{0}, y \in g^{-1}(A)}|x-y|$. Taking inf in (2.17) over all $x \in\left|\gamma_{k}\right|$ and $\omega \in A$, we obtain that

$$
\rho\left(g_{m_{k}}\left(\left|\gamma_{k}\right|\right), A\right):=\inf _{x \in g_{m_{k}}\left(\left|\gamma_{k}\right|\right), y \in A} \rho(x, y)>\varepsilon, \quad \forall k=1,2, \ldots
$$

We now show that there exists $\varepsilon_{1}>0$ such that

$$
\operatorname{dist}\left(g_{m_{k}}\left(\left|\gamma_{k}\right|\right), A\right)>\varepsilon_{1}, \quad \forall k=1,2, \ldots,
$$

where dist, as usual, denotes the Euclidean distance between the sets $A, B \subset \mathbf{R}^{n}$. Indeed, let (2.19) be violated, then for the number $\varepsilon_{l}=1 / l, l=1,2, \ldots$ there are $\xi_{l} \in\left|\gamma_{k_{l}}\right|$ and $\zeta_{l} \in A$ such that

$$
\left|g_{m_{k_{l}}}\left(\xi_{l}\right)-\zeta_{l}\right|<1 / l, \quad l=1,2, \ldots
$$

Without loss of generality, we may assume that the sequence $k_{l}, l=1,2, \ldots$, is increasing. Since $A$ is compact, we may assume that the sequence $\zeta_{l}$ converges to $\zeta_{0} \in A$ as $l \rightarrow \infty$. By the triangle inequality and from (2.20) it follows that

$$
\left|g_{m_{k_{l}}}\left(\xi_{l}\right)-\zeta_{0}\right| \rightarrow 0, \quad l \rightarrow \infty
$$

On the other hand, we recall that $\rho\left(g_{m_{k}}(x), \omega\right)=\left|g^{-1}\left(g_{m_{k}}(x)\right)-g^{-1}(\omega)\right|$, where $g: D_{0} \rightarrow D$ is some quasiconformal mapping of $D_{0}$ onto $D$, see (1.2). In particular, $g^{-1}$ is continuous in $D$, therefore, by the triangle inequality and (2.21), we obtain that

$$
\begin{aligned}
\left|g^{-1}\left(g_{m_{k_{l}}}\left(\xi_{l}\right)\right)-g^{-1}\left(\zeta_{l}\right)\right| \leqslant & \left|g^{-1}\left(g_{m_{k_{l}}}\left(\xi_{l}\right)\right)-g^{-1}\left(\zeta_{0}\right)\right| \\
& +\left|g^{-1}\left(\zeta_{0}\right)-g^{-1}\left(\zeta_{l}\right)\right| \rightarrow 0, \quad l \rightarrow \infty .
\end{aligned}
$$

However, by definition $\rho$ and from (2.22) it follows that

$$
\rho\left(g_{m_{k_{l}}}\left(\left|\gamma_{k_{l}}\right|\right), A\right) \leqslant \rho\left(g_{m_{k_{l}}}\left(\xi_{l}\right), \zeta_{l}\right)=\left|g^{-1}\left(g_{m_{k_{l}}}\left(\xi_{l}\right)\right)-g^{-1}\left(\zeta_{l}\right)\right| \rightarrow 0, \quad l \rightarrow \infty,
$$

which contradicts (2.18). The resulting contradiction indicates the validity of (2.19). 
We cover the continuum $A$ with the help of balls $B\left(x, \varepsilon_{1} / 4\right), x \in A$. Since $A$ is a compact set, we may assume that $A \subset \bigcup_{i=1}^{M_{0}} B\left(x_{i}, \varepsilon_{1} / 4\right), x_{i} \in A, i=1,2, \ldots, M_{0}$, $1 \leqslant M_{0}<\infty$. By definition, $M_{0}$ depends only on $A$, in particular, $M_{0}$ does non depend on $k$. We set

$$
\Gamma_{k}:=\Gamma\left(A, g_{m_{k}}\left(\left|\gamma_{k}\right|\right), D\right)
$$

Note that

$$
\Gamma_{k}=\bigcup_{i=1}^{M_{0}} \Gamma_{k i},
$$

where $\Gamma_{k i}$ consists of all paths $\gamma:[0,1] \rightarrow D$, belonging to the family $\Gamma_{k}$, such that $\gamma(0) \in B\left(x_{i}, \varepsilon_{1} / 4\right)$ and $\gamma(1) \in g_{m_{k}}\left(\left|\gamma_{k}\right|\right)$. We now show that

$$
\Gamma_{k i}>\Gamma\left(S\left(x_{i}, \varepsilon_{1} / 4\right), S\left(x_{i}, \varepsilon_{1} / 2\right), D\right) \text {. }
$$

Indeed, let $\gamma \in \Gamma_{k i}$, in other words, $\gamma:[0,1] \rightarrow D, \gamma(0) \in B\left(x_{i}, \varepsilon_{1} / 4\right)$ and $\gamma(1) \in$ $g_{m_{k}}\left(\left|\gamma_{k}\right|\right)$. By (2.19), $|\gamma| \cap B\left(x_{i}, \varepsilon_{1} / 4\right) \neq \varnothing \neq|\gamma| \cap\left(D \backslash B\left(x_{i}, \varepsilon_{1} / 4\right)\right)$. Therefore, by [Ku, Theorem 1.I.5.46] there is $0<t_{1}<1$ with the condition $\gamma\left(t_{1}\right) \in S\left(x_{i}, \varepsilon_{1} / 4\right)$. We can assume that $\gamma(t) \notin B\left(x_{i}, \varepsilon_{1} / 4\right)$ for $t>t_{1}$. Put $\gamma_{1}:=\left.\gamma\right|_{\left[t_{1}, 1\right]}$. By (2.19), $\left|\gamma_{1}\right| \cap B\left(x_{i}, \varepsilon_{1} / 2\right) \neq \varnothing \neq\left|\gamma_{1}\right| \cap\left(D \backslash B\left(x_{i}, \varepsilon_{1} / 2\right)\right)$. Thus, by [Ku, Theorem 1.I.5.46] there is $t_{1}<t_{2}<1$ with $\gamma\left(t_{2}\right) \in S\left(x_{i}, \varepsilon_{1} / 2\right)$. We may assume that $\gamma(t) \in B\left(x_{i}, \varepsilon_{1} / 2\right)$ for $t<t_{2}$. Put $\gamma_{2}:=\left.\gamma\right|_{\left[t_{1}, t_{2}\right]}$. Then, the path $\gamma_{2}$ is a subpath of $\gamma$, which belongs to the family $\Gamma\left(S\left(x_{i}, \varepsilon_{1} / 4\right), S\left(x_{i}, \varepsilon_{1} / 2\right), D\right)$. Thus, the relation $(2.25)$ is established.

Further reasoning is based, as before, on the successful choice of an admissible function $\eta$. Put

$$
\eta(t)= \begin{cases}4 / \varepsilon_{1}, & t \in\left[\varepsilon_{1} / 4, \varepsilon_{1} / 2\right], \\ 0, & t \notin\left[\varepsilon_{1} / 4, \varepsilon_{1} / 2\right] .\end{cases}
$$

Note that $\eta$ satisfies (1.4) for $r_{1}=\varepsilon_{1} / 4$ and $r_{2}=\varepsilon_{1} / 2$. Then, according to the definition of a ring $Q$-homeomorphism at $x_{i}$, we obtain that

$$
M\left(f_{m_{k}}\left(\Gamma\left(S\left(x_{i}, \varepsilon_{1} / 4\right), S\left(x_{i}, \varepsilon_{1} / 2\right)\right), D\right)\right) \leqslant\left(4 / \varepsilon_{1}\right)^{n} \cdot\|Q\|_{1}<c<\infty,
$$

where $c$ is some positive constant and $\|Q\|_{1}$ is $L_{1}$-norm of the function $Q$ in $D$. By (2.24), (2.25) and (2.26), using the subadditivity of modulus, we obtain that

$$
M\left(f_{m_{k}}\left(\Gamma_{k}\right)\right) \leqslant \frac{4^{n} M_{0}}{\varepsilon_{1}^{n}} \int_{D} Q(x) d m(x) \leqslant c \cdot M_{0}<\infty .
$$

Let us show that the estimate (2.27) contradicts the condition of the weak flatness of the boundary of the domain $D^{\prime}$. Let $U:=B_{q}\left(y_{0}, r_{0}\right)=\left\{y \in \overline{\mathbf{R}^{n}}: q\left(y, y_{0}\right)<r_{0}\right\}$, where $0<r_{0}<\min \left\{\delta / 4, m_{0} / 4\right\}, \delta$ is the number from the condition of the lemma and $q\left(K_{0}\right)=m_{0}$. (Here, as usual, $q\left(K_{0}\right)$ denotes the chordal diameter of the set $E=K_{0}$, defined by the formula (1.6)). Note that $\left|\gamma_{k}\right| \cap U \neq \varnothing \neq\left|\gamma_{k}\right| \cap\left(D^{\prime} \backslash U\right)$ for sufficiently large $k \in \mathbf{N}$, since $q\left(\left|\gamma_{k}\right|\right)>m_{0} / 2>m_{0} / 4$ by (2.16), in addition, $\overline{y_{k}} \in\left|\gamma_{k}\right|$ and $\overline{y_{k}} \rightarrow y_{0}$ as $k \rightarrow \infty$. Similarly, $f_{m_{k}}(A) \cap U \neq \varnothing \neq f_{m_{k}}(A) \cap\left(D^{\prime} \backslash U\right)$. Since $\left|\gamma_{k}\right|$ and $f_{m_{k}}(A)$ are continua, we obtain that

$$
f_{m_{k}}(A) \cap \partial U \neq \varnothing, \quad\left|\gamma_{k}\right| \cap \partial U \neq \varnothing,
$$

see [Ku, Theorem 1.I.5.46]. For a given $P:=c \cdot M_{0}>0$, where $c$ and $M_{0}$ is from (2.27), let $V \subset U$ be a neighborhood of the point $y_{0}$, corresponding to the definition of a weakly flat boundary. Then we have that

$$
M\left(\Gamma\left(E, F, D^{\prime}\right)\right)>c \cdot M_{0}
$$


for any continua $E, F \subset D^{\prime}$ with $E \cap \partial U \neq \varnothing \neq E \cap \partial V$ and $F \cap \partial U \neq \varnothing \neq F \cap \partial V$. Observe that

$$
f_{m_{k}}(A) \cap \partial V \neq \varnothing, \quad\left|\gamma_{k}\right| \cap \partial V \neq \varnothing
$$

for sufficiently large $k \in \mathbf{N}$. Indeed, $\overline{y_{k}} \in\left|\gamma_{k}\right|, x_{k} \in f_{m_{k}}(A)$, where $x_{k}, \overline{y_{k}} \rightarrow y_{0} \in V$ as $k \rightarrow \infty$. Therefore, $\left|\gamma_{k}\right| \cap V \neq \varnothing \neq f_{m_{k}}(A) \cap V$ for large $k \in \mathbf{N}$. In addition, we have that $q(V) \leqslant q(U) \leqslant 2 r_{0}<m_{0} / 2$. By (2.16), $q\left(\left|\gamma_{k}\right|\right)>m_{0} / 2$, therefore, $\left|\gamma_{k}\right| \cap\left(D^{\prime} \backslash V\right) \neq \varnothing$. Thus, by [Ku, Theorem 1.I.5.46], $\left|\gamma_{k}\right| \cap \partial V \neq \varnothing$. Similarly, $q(V) \leqslant q(U) \leqslant 2 r_{0}<\delta / 2$. Since $q\left(f_{m_{k}}(A)\right)>\delta$, we obtain that $f_{m_{k}}(A) \cap\left(D^{\prime} \backslash V\right) \neq$ $\varnothing$. By [Ku, Theorem 1.I.5.46], we have that $f_{m_{k}}(A) \cap \partial V \neq \varnothing$. Thus, relations (2.30) are established.

By (2.28), (2.29) and (2.30), we obtain that

$$
M\left(\Gamma\left(f_{m_{k}}(A),\left|\gamma_{k}\right|, D^{\prime}\right)\right)>c \cdot M_{0} .
$$

Note that $\Gamma\left(f_{m_{k}}(A),\left|\gamma_{k}\right|, D^{\prime}\right)=f_{m_{k}}\left(\Gamma\left(A, g_{m_{k}}\left(\left|\gamma_{k}\right|\right), D\right)\right)=f_{m_{k}}\left(\Gamma_{k}\right)$. Therefore, the relation $(2.31)$ can be written as

$$
M\left(\Gamma\left(f_{m_{k}}(A), g_{m_{k}}\left(\left|\gamma_{k}\right|\right), D\right)\right)=M\left(f_{m_{k}}\left(\Gamma_{k}\right)\right)>c \cdot M_{0}
$$

The relation obtained above contradicts the estimate (2.27). The resulting contradiction means that the above assumption $q\left(f_{m_{k}}(A), \partial D^{\prime}\right)<1 / k$ was incorrect. The proof of the lemma is complete.

\section{Proof of Theorem 1.7}

For the continuous extension of the mapping $h \in \mathfrak{S}_{\delta, A, Q}\left(D, D^{\prime}\right)$ to the boundary of the domain $D^{\prime}$, see Remark 1.8. The equicontinuity of $\mathfrak{S}_{\delta, A, Q}\left(D, D^{\prime}\right)$ at inner points of $D^{\prime}$ is the result of [SevSkv , Theorem 1.1].

We show the equicontinuity $\mathfrak{S}_{\delta, A, Q}\left(\bar{D}_{P}, \overline{D^{\prime}}\right)$ on $\partial D^{\prime}$. We carry out the proof by contradiction. Suppose there are a point $z_{0} \in \partial D^{\prime}$, a number $\varepsilon_{0}>0$, sequences $z_{m} \in \overline{D^{\prime}}$ and $\bar{h}_{m} \in \mathfrak{S}_{\delta, A, Q}\left(\bar{D}_{P}, \overline{D^{\prime}}\right)$ such that $z_{m} \rightarrow z_{0}$ as $m \rightarrow \infty$ and, in addition,

$$
\rho\left(\bar{h}_{m}\left(z_{m}\right), \bar{h}_{m}\left(z_{0}\right)\right) \geqslant \varepsilon_{0}, \quad m=1,2, \ldots,
$$

where $\rho$ is one of the metrics in $\bar{D}_{P}$, defined by the formula (1.2). Since $h_{m}=\left.\bar{h}_{m}\right|_{D^{\prime}}$ extends by continuity to the boundary of $D^{\prime}$, we may assume that $z_{m} \in D^{\prime}$ and, in addition, there is another sequence $z_{m}^{\prime} \in D^{\prime}, z_{m}^{\prime} \rightarrow z_{0}$ as $m \rightarrow \infty$, such that $\rho\left(h_{m}\left(z_{m}^{\prime}\right), \bar{h}_{m}\left(z_{0}\right)\right) \rightarrow 0$ as $m \rightarrow \infty$. Then from (3.1) it follows that

$$
\rho\left(h_{m}\left(z_{m}\right), h_{m}\left(z_{m}^{\prime}\right)\right) \geqslant \varepsilon_{0} / 2, \quad m \geqslant m_{0} .
$$

Since the domain $D$ is regular, the space $\bar{D}_{P}$ is compact. Therefore, we may assume that the sequences $h_{m}\left(z_{m}\right)$ è $\bar{h}_{m}\left(z_{0}\right)$ converge as $m \rightarrow \infty$ to some elements $P_{1}, P_{2} \in$ $\bar{D}_{P}, P_{1} \neq P_{2}$. Let $d_{m}$ and $g_{m}$ be sequences of descending domains corresponding to prime ends $P_{1}$ and $P_{2}$, respectively. By [IS, Lemma 3.1], cf. [KR 2 , Lemma 1], we may consider that the cuts $\sigma_{m}$ corresponding to domains $d_{m}, m=1,2, \ldots$, belong to spheres $S\left(\overline{x_{0}}, r_{m}\right)$ so that $\overline{x_{0}} \in \partial D$ and $r_{m} \rightarrow 0$ as $m \rightarrow \infty$. Choose $x_{0}, y_{0} \in A$ so that $x_{0} \neq y_{0}$ and $x_{0} \neq P_{1} \neq y_{0}$, where the continuum $A \subset D$ is from conditions of Theorem 1.7. Without loss of generality, we may assume that $d_{1} \cap g_{1}=\varnothing$ and $x_{0}, y_{0} \notin d_{1} \cup g_{1}$.

By Lemmas 2.3 and 2.10, there are disjoint paths $\gamma_{1, m}:[0,1] \rightarrow D$ and $\gamma_{2, m}$ : $[0,1] \rightarrow D$, the number $M_{0}>0$ and the number $N>0$ such that $\gamma_{1, m}(0)=x_{0}$, 
$\gamma_{1, m}(1)=h_{m}\left(z_{m}\right), \gamma_{2, m}(0)=y_{0}, \gamma_{2, m}(0)=h_{m}\left(z_{m}^{\prime}\right)$, wherein,

$$
M\left(f_{m}\left(\Gamma_{m}\right)\right) \leqslant N, \quad m \geqslant M_{0},
$$

where $f_{m}:=h_{m}^{-1}, \Gamma_{m}:=\Gamma\left(\left|\gamma_{1, m}\right|,\left|\gamma_{2, m}\right|, D\right)$ (see Figure 6).

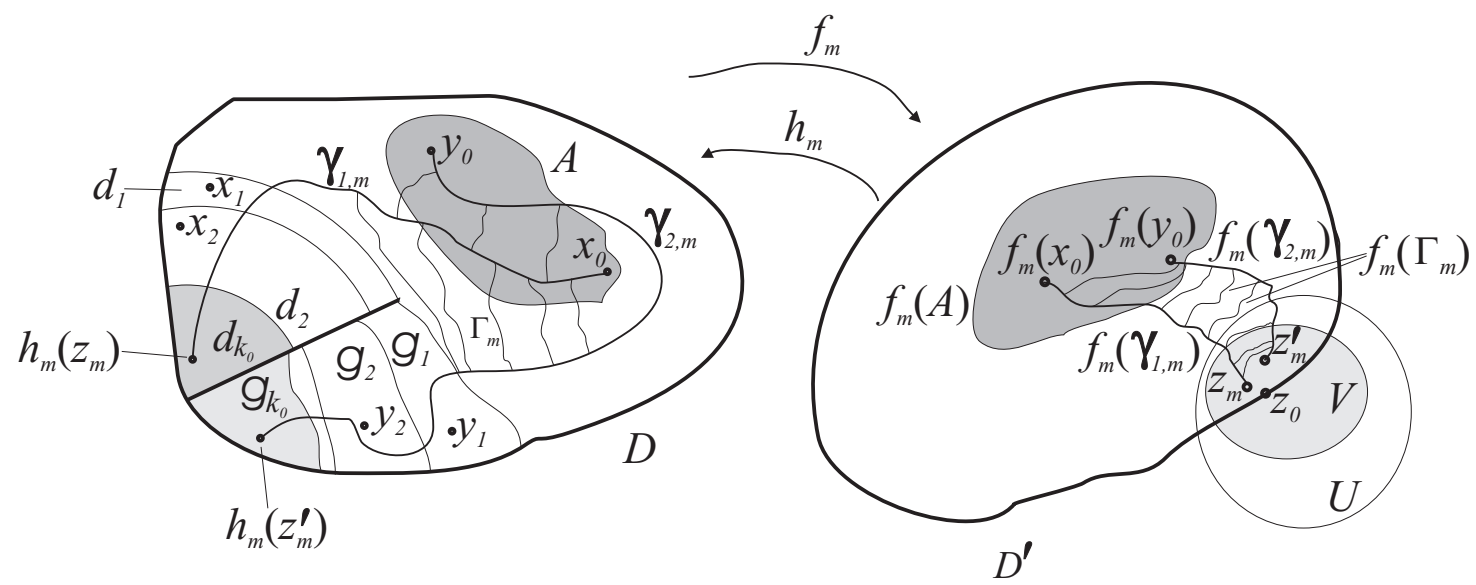

Figure 6. To the proof of Theorem 1.7.

On the other hand, by Lemma 2.13 there is a number $\delta_{1}>0$ such that $q\left(f_{m}(A)\right.$, $\left.\partial D^{\prime}\right)>\delta_{1}>0, m=1,2, \ldots$. From this we obtain that

$$
\begin{aligned}
& q\left(f_{m}\left(\left|\gamma_{1, m}\right|\right)\right) \geqslant q\left(z_{m}, f_{m}\left(x_{0}\right)\right) \geqslant(1 / 2) \cdot q\left(f_{m}(A), \partial D^{\prime}\right)>\delta_{1} / 2, \\
& q\left(f_{m}\left(\left|\gamma_{2, m}\right|\right)\right) \geqslant q\left(z_{m}^{\prime}, f_{m}\left(y_{0}\right)\right) \geqslant(1 / 2) \cdot q\left(f_{m}(A), \partial D^{\prime}\right)>\delta_{1} / 2
\end{aligned}
$$

for large $m \in \mathbf{N}$. Choose a chordal ball $U:=B_{q}\left(z_{0}, r_{0}\right)$, where $r_{0}>0$ and $r_{0}<$ $\delta_{1} / 4$, and $\delta_{1}$ is the number from relations (3.4). Note that $f_{m}\left(\left|\gamma_{1, m}\right|\right) \cap U \neq \varnothing \neq$ $f_{m}\left(\left|\gamma_{1, m}\right|\right) \cap\left(D^{\prime} \backslash U\right)$ for sufficiently large $m \in \mathbf{N}$, because $q\left(f_{m}\left(\left|\gamma_{1, m}\right|\right)\right) \geqslant \delta_{1} / 2$ and $z_{m} \in f_{m}\left(\left|\gamma_{1, m}\right|\right), z_{m} \rightarrow z_{0}$ as $m \rightarrow \infty$. Due to the same considerations $f_{m}\left(\left|\gamma_{2, m}\right|\right) \cap U \neq$ $\varnothing \neq f_{m}\left(\left|\gamma_{2, m}\right|\right) \cap\left(D^{\prime} \backslash U\right)$. Since $f_{m}\left(\left|\gamma_{1, m}\right|\right)$ and $f_{m}\left(\left|\gamma_{2, m}\right|\right)$ are continua, then by [Ku, Theorem 1.I.5.46]

$$
f_{m}\left(\left|\gamma_{1, m}\right|\right) \cap \partial U \neq \varnothing, \quad f_{m}\left(\left|\gamma_{2, m}\right|\right) \cap \partial U \neq \varnothing .
$$

For a fixed $P:=N>0$, where $N$ is from (3.3), let $V \subset U$ be a neighborhood of the point $z_{0}$, corresponding to the definition of a weakly flat boundary, that is, such that for any continua $E, F \subset D^{\prime}$ with $E \cap \partial U \neq \varnothing \neq E \cap \partial V$ and $F \cap \partial U \neq \varnothing \neq F \cap \partial V$ the inequality

$$
M\left(\Gamma\left(E, F, D^{\prime}\right)\right)>N
$$

holds. Note that for sufficiently large $m \in \mathbf{N}$

$$
f_{m}\left(\left|\gamma_{1, m}\right|\right) \cap \partial V \neq \varnothing, \quad f_{m}\left(\left|\gamma_{2, m}\right|\right) \cap \partial V \neq \varnothing .
$$

Indeed, $z_{m} \in f_{m}\left(\left|\gamma_{1, m}\right|\right)$ and $z_{m}^{\prime} \in f_{m}\left(\left|\gamma_{2, m}\right|\right)$, where $z_{m}, z_{m}^{\prime} \rightarrow z_{0} \in V$ as $m \rightarrow \infty$ Therefore, $f_{m}\left(\left|\gamma_{1, m}\right|\right) \cap V \neq \varnothing \neq f_{m}\left(\left|\gamma_{2, m}\right|\right) \cap V$ for large $m \in \mathbf{N}$. In addition, $q(V) \leqslant$ $q(U)=2 r_{0}<\delta_{1} / 2$ and since by $(3.4) q\left(f_{m}\left(\left|\gamma_{1, m}\right|\right)\right)>\delta_{1} / 2$, then $f_{m}\left(\left|\gamma_{1, m}\right|\right) \cap\left(D^{\prime} \backslash V\right) \neq$ $\varnothing$. Then $f_{m}\left(\left|\gamma_{1, m}\right|\right) \cap \partial V \neq \varnothing$ (see [Ku, Theorem 1.I.5.46]). Similarly, $q(V) \leqslant q(U)=$ $2 r_{0}<\delta_{1} / 2$ and, since by $(3.4) q\left(f_{m}\left(\left|\gamma_{2, m}\right|\right)\right)>\delta_{1} / 2$, then $f_{m}\left(\left|\gamma_{2, m}\right|\right) \cap\left(D^{\prime} \backslash V\right) \neq \varnothing$. Now, by $\left[\mathrm{Ku}\right.$, Theorem 1.I.5.46] we obtain that $f_{m}\left(\left|\gamma_{1, m}\right|\right) \cap \partial V \neq \varnothing$. Thus, (3.7) is proved.

According to (3.6) and taking into account (3.5) and (3.7), we obtain that

$$
M\left(f_{m}\left(\Gamma_{m}\right)\right)=M\left(\Gamma\left(f_{m}\left(\left|\gamma_{1, m}\right|\right), f_{m}\left(\left|\gamma_{2, m}\right|\right), D^{\prime}\right)\right)>N,
$$


which contradicts the inequality (3.3). The resulting contradiction indicates that the original assumption made in (3.1) is incorrect. The theorem is proved.

\section{Examples}

Example 4.1. Let $D$ be the unit square from which the sequence of segments $I_{k}=\left\{z=(x, y) \in \mathbf{R}^{2}: x=1 / k, 0<y<1 / 2\right\}, k=2,3, \ldots$, is removed (see Figure 7).

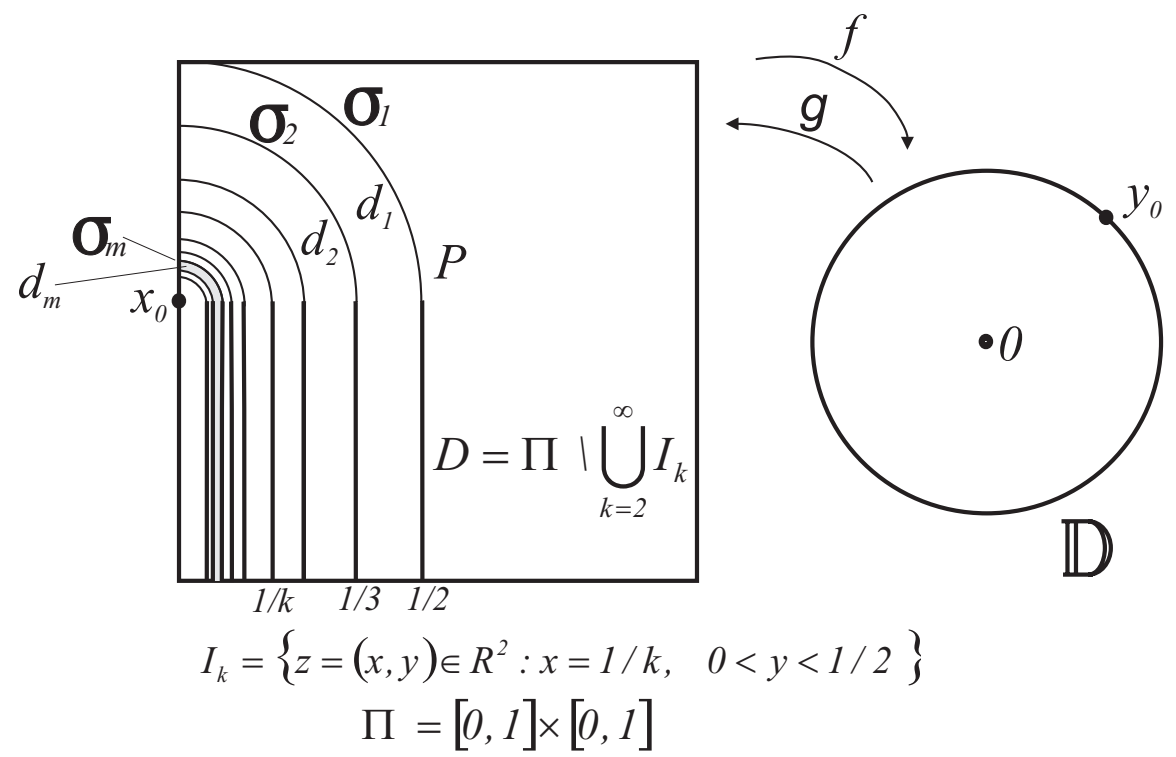

Figure 7. Illustration for Example 4.1.

Consider the prime end $P$ in the domain $D$, formed by cuts

$$
\sigma_{m}=\left\{z=x_{0}+\frac{e^{i \varphi}}{m+1}, x_{0}=(0,1 / 2), 0 \leqslant \varphi \leqslant \pi / 2\right\}, \quad m=1,2, \ldots, .
$$

It can be shown that the end $P$ is really prime. According to the Riemann mapping theorem, there exists a conformal mapping $g$ of the unit disk $\mathbf{D}$ onto the domain $D$ and by the Caratheodory theorem, a prime end $P$ corresponds to some point $y_{0} \in \partial \mathbf{D}$ so that $C\left(f, y_{0}\right)=I(P), f=g^{-1}$, see [CL, Theorem 9.4]. It follows that we may choose two sequences $z_{k}, w_{k} \in D, k=1,2, \ldots$, such that $z_{k}, w_{k} \rightarrow P, z_{k} \rightarrow z_{0}$ and $w_{k} \rightarrow w_{0}$ as $k \rightarrow \infty, z_{0} \neq w_{0}$, while $f\left(z_{k}\right) \rightarrow y_{0}$ and $f\left(w_{k}\right) \rightarrow y_{0}$ as $k \rightarrow \infty$. Consequently, the mapping $f:=g^{-1}$ does not have a continuous extension to the point $y_{0}$ in the pointwise sense, although $g$ has a continuous extension $\bar{g}: \overline{\mathbf{D}} \rightarrow \bar{D}_{P}$.

Consider another auxiliary family of mappings. As is known, linear fractional automorphisms of the unit disk $\mathbf{D} \subset \mathbf{C}$ have the form

$$
f(z)=e^{i \theta} \frac{z-a}{1-\bar{a} z}, \quad z \in \mathbf{D}, \quad a \in \mathbf{D}, \quad \theta \in[0,2 \pi) .
$$

We set, for example, $\theta=0, a=1 / n, n=1,2, \ldots$. In this case, consider the family of mappings $\widetilde{f}_{n}(z)=\frac{z-1 / n}{1-z / n}=\frac{n z-1}{n-z}$. Let $\widetilde{A}=[0,1 / 2]$. Then we obtain that $\widetilde{f}_{n}(0)=-1 / n \rightarrow 0$ and $\widetilde{f}_{n}(1 / 2)=\frac{n-2}{2 n-1} \rightarrow 1 / 2$ as $n \rightarrow \infty$. Thus, the mappings $\widetilde{f}_{n}$ satisfy the condition $q\left(\widetilde{f}_{n}(\widetilde{A})\right) \geqslant \delta$ say, with $\delta=1 / 4$.

Now we put $f_{n}:=\widetilde{f}_{n} \circ f$. Note that the mappings $f_{n}$ are conformal; therefore, they satisfy the estimate (1.3) for $Q \equiv 1$ at each point $x_{0} \in \bar{D}$, see [Pol, Theorem 1], cf. [MRSY 1 , Theorems 4.6 and 6.10] and [MRSY ${ }_{3}$, Theorems 8.1 and 8.6]. Note 
also that the mappings $f_{n}$ satisfy all the conditions of Theorem 1.7, in particular, $q\left(f_{n}(A)\right) \geqslant \delta$ with $\delta=1 / 4$, where $A:=f^{-1}(\widetilde{A})$. Note that the unit disk $\mathbf{D}$ has a weakly flat boundary by $\left[\mathrm{Va}_{1}\right.$, Theorems 17.10 and 17.12]. By construction, mappings $g_{n}:=f_{n}^{-1}$ do not even have a pointwise continuous extension to $\partial \mathbf{D}$ in particular, the family of these mappings is not equicontinuous in $\bar{D}$. Nevertheless, the extended family $\bar{g}_{n}: \overline{\mathbf{D}} \rightarrow \bar{D}_{P}$ is equicontinuous in terms of prime ends by Theorem 1.7.

Put now $\theta=0, a=(n-1) / n, n=1,2, \ldots$ and $\widetilde{f}_{n}^{-1}(z)=\frac{z-(n-1) / n}{1-z(n-1) / n}=\frac{n z-n+1}{n-n z+1}$. We set $f_{n}:=\tilde{f}_{n} \circ f$. It is easy to understand that the sequence $\tilde{f}_{n}^{-1}$ is locally uniformly converges to a constant function -1 in the unit disk. On the other hand, we have the equality $\widetilde{f}_{n}^{-1}(1)=1$, which immediately implies that the sequence $\widetilde{f}_{n}^{-1}$ is not equicontinuous at the point 1 .

It follows that the sequence $f_{n}^{-1}$ is also not equicontinuous at point 1 . The reason for this is a violation of the requirement $q\left(f_{n}(A)\right) \geqslant \delta$.

Example 4.2. It is also easy to indicate a similar example of a family of mappings with unbounded characteristic. Let $D$ be the domain constructed in Example 4.1. Then we put $f_{1}(z)=\frac{1}{e \sqrt{2}}|z-1 / 2|$. Note that $f_{1}$ maps $D$ onto a domain $D_{1}$ lying in the ball $B(0,1 / e)$. Now we put $f_{2}(z):=\frac{z}{|z| \log \frac{1}{|z|}}$ and $F(z)=\left(f_{2} \circ f_{1}\right)(z)$. Using the technique outlined in the consideration of [MRSY ${ }_{3}$, Proposition 6.3], we may establish that $F$ is a ring $Q$-homeomorphism in $\bar{D}$ with $Q(z)=\log \frac{e \sqrt{2}}{|z-1 / 2|}$. One can also prove that $Q \in L^{1}(D)$. Note that $D_{1}$ is a simply connected domain, therefore, by the Riemann theorem, it is possible to map it onto the unit disk using some conformal mapping $f_{3}$.

Consider the family of mappings $\widetilde{f}_{n}(z)=\frac{z-1 / n}{1-z / n}=\frac{n z-1}{n-z}$. We set $F_{n}(z)=\left(\widetilde{f}_{n}\right.$ 。 $\left.f_{3} \circ f_{2} \circ f_{1}\right)(z)$. Let $\widetilde{A}=[0,1 / 2]$. Then we obtain that $\tilde{f}_{n}(0)=-1 / n \rightarrow 0$ and $\widetilde{f}_{n}(1 / 2)=\frac{n-2}{2 n-1} \rightarrow 1 / 2$ as $n \rightarrow \infty$. Thus, the mappings $\widetilde{f}_{n}$ satisfy the condition $q\left(\widetilde{f}_{n}(\widetilde{A})\right) \geqslant \delta$ say, with $\delta=1 / 4$. In this case, the mappings $F_{n}$ satisfy the condition $q\left(F_{n}(A)\right) \geqslant \delta$ say, with $\delta=1 / 4$ and $A=\left(f_{1}^{-1} \circ f_{2}^{-1} \circ f_{3}^{-1}\right)(\widetilde{A})$.

Since the modulus of families of paths does not change under conformal transformations, the mappings $F_{n}$ are ring $Q$-maps in $D$, where $Q(z)=\log \frac{e \sqrt{2}}{|z-1 / 2|}$ (see $\left[\mathrm{Va}_{1}\right.$, Theorem 8.1]). The mappings $G_{n}=F_{n}^{-1}$ do not have a pointwise continuous extension to $\partial \mathbf{D}$, however, this extension is valid in the sense of prime ends. In addition, the family of extended mappings $\bar{G}_{n}: \overline{\mathbf{D}} \rightarrow \bar{D}_{P}, n=1,2, \ldots$, is equicontinuous in $\overline{\mathbf{D}}$ by Theorem 1.7 .

Example 4.3. As it was stated at the beginning of the article, let us give an example of a family of homeomorphisms, in which the transition to the inverse family takes us beyond the limits of the class of mappings under consideration. At the same time, it is also an example of a family that is equicontinuous in the closure of an domain, however, the inverse of it is not such. We will identify an isolated point of the boundary of a domain with a prime end; in this case, we will consider some descending sequence of spheres with a center at this point as cuts. Note that the example with minor modifications was taken from our earlier paper [SSI].

Let $n \geqslant 2$ and let $p \geqslant 1$ be a number, such that $n / p(n-1)<1$. Put $\alpha \in$ $(0, n / p(n-1))$. Let $\mathbf{B}^{n}=\left\{x \in \mathbf{R}^{n}:|x|<1\right\}$. We define a sequence of mappings $f_{m}$ 
of $\mathbf{B}^{n}$ onto $B(0,2)$ in the following way:

$$
f_{m}(x)= \begin{cases}\frac{1+|x|^{\alpha}}{|x|} \cdot x, & 1 / m \leqslant|x| \leqslant 1 \\ \frac{1+(1 / m)^{\alpha}}{(1 / m)} \cdot x, & 0<|x|<1 / m\end{cases}
$$

see Figure 8.

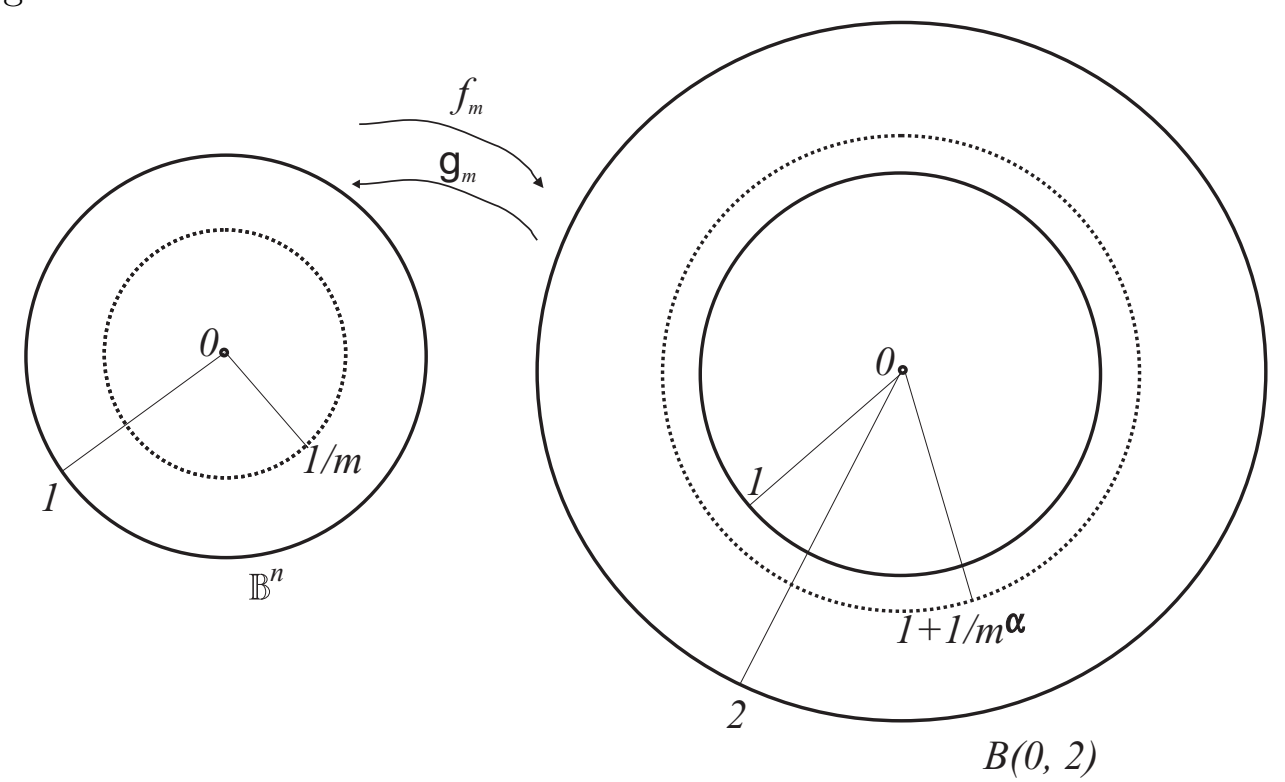

Figure 8. Illustration for Example 4.3.

Notice, that $f_{m}$ satisfies (1.3) in $\overline{\mathbf{B}^{n}}$ for $Q=\left(\frac{1+|x|^{\alpha}}{\alpha|x|^{\alpha}}\right)^{n-1} \in L^{p}\left(\mathbf{B}^{n}\right)$ at every $x_{0} \in \overline{\mathbf{B}^{n}}$. As above, this can be shown by the reasoning used in the consideration of [MRSY ${ }_{3}$, Proposition 6.3]. By [Va ${ }_{1}$, Theorems 17.10 and 17.12] B(0,2) has a weakly flat boundary, cf. [Vu, Lemma 4.3]. Observe that $f_{m}$ fixes an infinite number of points of the unit ball for all $m \geqslant 2$, so that the condition $q\left(f_{m}(A)\right) \geqslant \delta$ holds for some continuum $A \subset D$ and some $\delta>0$. By direct computation, it can be established that the family $\overline{\mathfrak{G}}=\left\{\bar{g}_{m}\right\}_{m=1}^{\infty}, g_{m}:=f_{m}^{-1}$, consisting of all extended mappings $\bar{g}_{m}: \overline{B(0,2)} \rightarrow \overline{\mathbf{B}^{\mathbf{n}}}$, is equicontinuous in $\overline{B(0,2)}$.

Observe that the "inverse" family $F=\left\{\bar{f}_{m}\right\}_{m=1}^{\infty}, \bar{f}_{m}: \overline{\mathbf{B}^{n}} \rightarrow \overline{B(0,2)}$, is not equicontinuous in $\overline{\mathbf{B}^{n}}$. In fact, $\left|f_{m}\left(x_{m}\right)-f(0)\right|=1+1 / m^{\alpha} \not \rightarrow 0$ as $m \rightarrow \infty$, where $\left|x_{m}\right|=1 / m$. The reason for the last circumstance is that $g_{m}$ are not ring $Q$-homeomorphism with some integrable $Q$ in $B(0,2)$. Indeed, if the latter were true, then the family of mappings $F=\left\{f_{m}\right\}_{m=1}^{\infty}$ would be equicontinuous in $\overline{\mathbf{B}^{n}}$ by see [SSI, Theorem 1].

\section{References}

[CL] Collingwood, E. F., and A. J. LohwateR: The theory of cluster sets. - Cambridge Tracts in Math. and Math. Physics 56, Cambridge Univ. Press, Cambridge, 1966.

[Cr] Cristea, M.: Open discrete mappings having local $A C L^{n}$ inverses. - Complex Var. Elliptic Equ. 55:1-3, 2010, 61-90.

[GRY] Gutlyanski, V., V. Ryazanov, and E. Yakubov: The Beltrami equations and prime ends. - Ukr. Mat. Visn. 12:1, 2015, 27-66 (in Russian); English transl. in J. Math. Sci. (N.Y.) 210:1, 2015, 22-51.

[HK] Herron, D., and P. Koskela: Quasiextremal distance domains and conformal mappings onto circle domains. - Complex Var. Theory Appl. 15, 1990, 167-179. 
[IS] Ilyutko, D. P., and E. A. Sevost'yanov: On prime ends on Riemannian manifolds. J. Math. Sci. 241:1, 2019, 47-63.

[KR 1 Kovtonyuk, D. A., and V. I. RyazAnov: On the theory of prime ends for space mappings. - Ukr. Mat. Zh. 67:4, 2015, 467-479 (in Russian); English transl. in Ukrainian Math. J. 67:4, 2015, 528-541.

$\left[\mathrm{KR}_{2}\right]$ Kovtonyuk, D. A., and V.I. Ryazanov: Prime ends and Orlicz-Sobolev classes. Algebra i Analiz 27:5, 2015, 81-116 (in Russian); English transl. in St. Petersburg Math. J. 27:5, 2016, 765-788.

[Ku] Kuratowski, K.: Topology. Volume 2. - Academic Press, New York-London, 1968.

[MRV] Martio, O., S. Rickman, and J. VÄIsÄLÄ: Distortion and singularities of quasiregular mappings. - Ann. Acad. Sci. Fenn. Ser. A I Math. 465, 1970, 1-13.

[MRSY ${ }_{1}$ ] Martio, O., V. Ryazanov, U. Srebro, and E. Yakubov: Mappings with finite length distortion. - J. Anal. Math. 93, 2004, 215-236.

[MRSY ${ }_{2}$ ] Martio, O., V. Ryazanov, U. Srebro, and E. Yakubov: On $Q$-homeomorphisms. Ann. Acad. Sci. Fenn. Math. 30:1, 2005, 49-69.

[MRSY ${ }_{3}$ ] Martio, O., V. Ryazanov, U. Srebro, and E. Yakubov: Moduli in modern mapping theory. - Springer Science + Business Media, LLC, New York, 2009.

[Na] NÄKkI, R.: Prime ends and quasiconformal mappings. - J. Anal. Math. 35, 1979, 13-40.

[NP 1 NÄKKI, R., and B. PALKA: Uniform equicontinuity of quasiconformal mappings. - Proc. Amer. Math. Soc. 37:2, 1973, 427-433.

$\left[\mathrm{NP}_{2}\right] \quad \mathrm{NÄKKI}, \mathrm{R}$., and B. PALKA: Boundary regularity and the uniform convergence of quasiconlormal mappings. - Comment. Math. Helvetici 54, 1979, 458-476.

[Pol] PoletskiI, E. A.: The modulus method for non-homeomorphic quasiconformal mappings. - Mat. Sb. 83:2, 1970, 261-272 (in Russian).

[RSY] Ryazanov, V., U. SRebro, and E. Yakubov: On ring solutions of Beltrami equations. - J. Anal. Math. 96, 2005, 117-150.

[SalSev] Salimov, R. R., and E. A. Sevost'yanov: On the equicontinuity of one family of inverse mappings in terms of prime ends. - Ukr. Math. Zh. 70:9, 2018, 1264-1273 (in Russian); English transl. in Ukr. Math. J. 70:9, 2019, 1456-1466.

[Sev] SevosT'Yanov, E. A.: On boundary extension of mappings in metric spaces in the terms of prime ends. - Ann. Acad. Sci. Fenn. Math. 44:1, 2019, 65-90.

[SevSkv 1 Sevost'Yanov, E. A., and S. A. Skvortsov: On the convergence of mappings in metric spaces with direct and inverse modulus conditions. - Ukr. Mat. Zh. 70:7, 2018, 952-967 (in Russian); English transl. in Ukr. Math. J. 70:7, 2018, 1097-1114.

[SevSkv 2 ] Sevost'yanov, E. A., and S. A. Skvortsov: On mappings whose inverse satisfy the Poletsky inequality. - Ann. Acad. Sci. Fenn. Math. 45, 2020, 259-277.

[SSI] Sevost'yanov, E. A., S. A. Skvortsov, and N. S. Ilkevych: On boundary behavior of mappings with two normalized conditions. - Mat. Studii 49:2, 2018, 150-157.

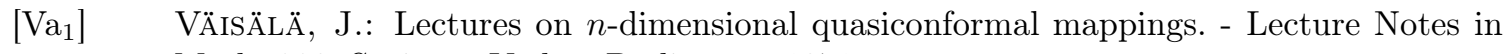
Math. 229, Springer-Verlag, Berlin etc., 1971.

[Va $\left.{ }_{2}\right]$ VÄIsÄLÄ, J.: Modulus and capacity inequalities for quasiregular mappings. - Ann. Acad. Sci. Fenn. Ser. A I Math. 509, 1972, 1-14.

[Vu] Vuorinen, M.: On the existence of angular limits of $n$-dimensional quasiconformal mappings. - Ark. Mat. 18, 1980, 157-180. 